NBER WORKING PAPER SERIES

FISCAL POLICY WITH IMPURE

INTERGENERATIONAL ALTRUISM

Andrew B. Abel

B. Douglas Bernheim

Working Paper No. 2613

NATIONAL BUREAU OF ECONOMIC RESEARCH

1050 Massachusetts Avenue

Cambridge, MA 02138

June 1988

This work is part of the NBER's program in Taxation and was supported by the National Science Foundation, through Grants No. SES 8408618 and SES8607630. We would like to thank seminar participants at the various presentations of this paper for helpful comments. Any views expressed here are those of the authors, and should not be attributed to any other individual or organization. 
NBER Working Paper \#2613

June 1988

\section{FISCAL POLICY WITH IMPURE \\ INTERGENERATIONAL ALTRUISM}

\section{ABSTRACT}

Recent work demonstrates that dynastic assurptions guantee the irrelevance of all redistributional policies, distortionary taxes, and prices--the neutrality of fiscal policy (Ricardian equivalence) is only the "tip of the iceburg." In this paper, we investigate the possibility of reinstating approximate Ricardian equivalence by introducing a-small amount of friction in intergenerational links. If Ricardian equivalence depends upon significantly shorter chains of links than do these stronger neutrality results, then friction may dissipate the effects that generate strong neutrality, without significantly affecting the Ricardian result. Although this intuition turns out to be essentially correct, we show that models with small amounts of friction have other untenable implications. We conclude that the theoretical case for Ricardian equivalence remins tenuous.

Professor Andrew B. Abel The Wharton School University of Pennsylvania Philadelphia, PA 19104
Professor B. Douglas Bernheim Department of Economics Stanford University Stanford, CA 94305 


\section{Introduction}

In recent years, Robert Barro's [1974] version of "Ricardian equivalence" has stimulated much controversy concerning the effects of government budget deficits and social security programs. In his wellknown paper, Barro supplemented the traditional overlapping generations model with intergenerational altruism, and argued, in essence, that voluntary transfers between parents and children cause the representative family to behave as though it is a single, infinite-lived individual-a "dynastic" unit. From the point of view of the family, neither debt nor social security alters available alternatives; both are therefore neutral. Thus, Barro's analysis identifies the strength of intergenerational altruism as a key factor in determining the effects of government bond issues and public pension programs.

Recently, Bernheir and Bgwell [1988] have argued against the applicability of Ricardian equivalence by denonstrating that Barro's assumptions guarantee the irrelevance of all redistributional policies, distortionary taxes, and prices--the neutrality of fiscal policy is only the "tip of the iceburg." Their results rely on the existence of intrafamily linkages, which arise whenever two unrelated individuals produce a common child. Bernheim and Eagwell concluded that, since the se other propositions do not hold even approximately, one cannot assert that the world is approximately dynastic. Accordingly, all conclusions following from the dynastic framework (including Ricardian equivalence) are suspect.

Bernheim and Begwell also noted that it might be possible to 
reinstate approximate Ricardian equivalence without generating untenable consequences by introducing a small amount of "friction". Intuitively, friction would cumlate with each link and would become substantial for long chains. Since Ricardian equivalence for debt redeened within a few generations) presumably depends on short chains while the bernheimBagrell results presumably depend on long ones (we note that these presumptions wa be erroneous--see section 7 ), the introduction of friction might just do the triok.

The purpose of this paper is to evaluate the preceding argument by formally introducing various forms of friction into a model with altruistically motivated intergenerational transfers. We focus on frictions arising from three sources: the derivation of pleasure directly from the act of giving; incomplete information about others' preferences; and egalitarian social norms that constrain parents to divide transfers eveniy between children. The first two sources of friction turn out to be quite similar analytically, and give rise to qualitatively similar results. In particular, one can obtain approximate Ricardian equivalence by introducing a sufficiently small amount of friction. Furthermore, for any given amount of friction, one can reingtate the relevance of other redistributional policies by taking the population to be sufficiently large (it follows from this that taxes w1l distort behavior, and prices will play an important allocational role). However, there is a hitch: by simultaneously taking friction to be small and population to be large, one drives each individual's marginal propensity to consume out of wealth to zero. In resolving 
several paradoxes posed by Bernheim and Bagwell, one therefore merely encounters another.

The introduction of egalitarian constraints generates some intruiging results. Most importantly, one obtains exact Ricardian equivalence in a world where other redistributional policies have significant allocative effects. Since there is no need to assume that this source of friction is "small," one does not encounter the ownweal th effect puzzle noted above. We are troubled, however by the rather ad hoc nature of this constraint. In addition, its imposition generates a new paradox: we show that an exogenous increase in the wealth of any given individual is never Pareto improving. Consequently, we conclude that the theoretical case for Ricardian equivalence remins tenuous even when one explicitly recognizes sources of economic friction.

We organize our discussion as follows. Section 2 lays out the basic model, describes an appropriate notion of equilibrium, and presents some technical results which facilitate the analysis of subsequent sections. In sections 3 through 6 we consider, respectively, specialized cases in which a) there is no friction, b) altruists derive utility in part directly from the act of giving, c) agents have incomplete information about each others' preferences, and d) parents are constrained to divide transfers equally between their children. Section 7 contains some concluding remarks. We defer all technical manipulions and proofs to the appendices. Appendix A contains a complete treatment of comparative statics for cases b and c above, while 
Appendix $E$ treats case $d$. We present proofs of specific results in Appendix $C$.

\section{The Mode 1}

We consider an economy comprised of $2 \mathrm{~N}$ households. Despite the fact that we treat each household as if it consists of a single individual, one should for the purpose of interpretation think of households as merried couples. The population is evenly divided between two groups of households, henceforth referred to as "parents" and "children." Thus, there are $N$ parents (labelled $\left.p_{i}, i=1, \ldots, N\right)$, and $N$ children (labeiled $\left.k_{i}, i=1, \ldots, N\right)$. Every parent has two children, and every child has two parents (reflecting the fact that spouses origirally come from different households). We assume in

particular that $p_{i}{ }^{\prime} s$ children are $k_{i}$ and $k_{i+1}$ (where, by convention, $\left.k_{N+1}=k_{1}\right)$. It is therefore appropriate to think of intrafamily relations as a kind of circle (pictured in figure 1), consisting of an outer layer (parents) and an inner layer (children). This representation of intrafamily relations is unquestionably highly stylized, and does not reflect the full complexity of family networks, particularly in cases where these networks span more than two generations (see Bernheim and Bagwell [1988]). On the other hand, this framework has the advantage of rendering our current analytic objectives tractable, while in all likelihood doing very little violence to the underlying economic issues. We retarn to this point in section 7 , and argue that more realistic modelling of family networks would only tend to strengthen our conclusions. 
Parent $P_{i}$ is endowed with wea:th, $W_{i} ;$ similarly, child $k_{i}$ is endowed with $w_{i}$. Parent $p_{i}$ divides his wealth between consumption $\left(C_{i}\right)$, a transfer to child $k_{i}\left(T_{i}\right)$, and a transfer to child $k_{i+1}\left(t_{i}\right)$,

$$
C_{i}=W_{i}-T_{i}-t_{i},
$$

subject, of course, to non-negativity constraints $\left(C_{i} \geq 0, T_{i} \geq 0\right.$, $\left.t_{i} \geq 0\right)$. Child $i$ receives transfers from parents $p_{i}$ and $p_{i-1}$ (where, by convention, $p_{0} \equiv p_{N}$ ), and consumes all available resources:

$$
c_{i}=w_{i}+T_{i}+t_{i-1}
$$

We suppose that children are completely selfish, so that the wellbeing of child $k_{i}$ is given by

$$
u_{i}=u\left(c_{i}\right)
$$

With probability $\pi$, parent $P_{i}$ is also completely selfish, so that his well being is given by

$$
u_{i}=u\left(C_{i}\right)
$$

(note that the felicity function for parents is identical to that for children--this restriction is inessential). With probability

$(1-\pi)$, parent $p_{i}$ is altruistic; this entails non-paternalistic altruism for his child (as in Berro [1974]), and possibly some concern for the magnitude of his bequests (as in Andreoni [1986]):

$$
u_{i}=u\left(c_{i}\right)+\beta\left[u\left(c_{i}\right)+u\left(c_{i+1}\right)\right]+\alpha\left[v\left(T_{i}\right)+v\left(t_{i}\right)\right]
$$


$(\alpha, \beta>0)$. For simplicity, we assume that the random events that determine parental preferences are distributed independently over pecents. Throughout, we also assume thet $u(\cdot)$ and $v(\cdot)$ are twice continuously differentiabie and strictly concave.

The final allocation of resources is determined through a simultaneous move game, in which each perent chooses his own consumption, as well as intergenerational transfers. Each parent's preferences are privete information; while perent $p_{1}$ knows whether or not he himself is altuistic, his information concerning others is limited to knowledge of the distribution of preferences described above. It is therefore necessary to employ a solution concept that allows for incomplete information. The natural choice is to focus attention on Bayesian Nash Equilibria (see Harsanyi [1967-68]).

In a Bayesian Nash Equilibrium (hencefort BNE), we assign to each parent a function mepping his preferences into decisions. These decisions must maximize his expected utility given associated preferences, and given the distribution of other parents' decisions induced by their assigned functions. In the current context, a BNE has a particularly simple form. When parent i is selfish, he will obviously set $T_{i}, t_{i}=0$, regardless of what other parents do. Thus, we need only describe the choices, $\left(T_{i}^{*}, t_{i}^{*}\right)$, which are contingent upon parent $i$ being altruistic. Accordingly, $\left(T_{i}^{*}, t_{i}^{*}\right)_{i=1}^{N}$ is a BNE if for all $1,\left(T_{i}^{*}, t_{i}^{*}\right)$ solves 
(3) $\max _{T_{i}, t_{i}} u\left(W_{i}-T_{i}-t_{i}\right)+\beta\left\{(1-x)\left[u\left(w_{i}+T_{i}+t_{i-1}^{*}\right)+u\left(w_{i+1}+T_{i+1}^{*}+t_{i}\right)\right]\right.$

$$
\left.+\pi\left[u\left(w_{i}+T_{i}\right)+u\left(w_{i+1}+t_{i}\right)\right]\right\}+\alpha\left[v\left(T_{i}\right)+v\left(t_{i}\right)\right]
$$

(subject to non-negativity constants). We note in passing that when $\pi$ equals either 0 or 1 (so that information is complete), this definition reduces to the more standard notion of a Nash equilibrium.

Throughout much of our analysis, we will assume that resources are initially distributed evenly within generations. That is,

$$
\begin{gathered}
w_{i}=w \\
w_{i}=w
\end{gathered}
$$

for all $i$. When we assume symmetric endowments, we will also focus attention on symmetric equilibria, which have the property that the magnitudes of all transfers (conditional upon the parent being altruistic) are identical (i.e., $T_{i}^{*}=t_{i}^{*}=T^{*}$ for ali $\left.i=1, \ldots, N\right)$. We now present three technical results which justify the comparative statics performed in subsequent sections. The first of these establishes existence.

Theorem 1: For all endowment profiles $\left(W_{i}, w_{i}\right)_{i=1}^{N}$ a ENE exists.

Next, we show that symmetric equilibria do indeed exist when endowents are symmetric.

Theorem 2: If endowments are distributed symmetrically, then there exists a symmetric BNE. Furthermore, the associated transfer, $\mathrm{T}^{*}$, 
is independent of $N$.

The second portion of this result establishes that the aliocation of resources is in some important sense independent of population size. This conclusion will feature prominently in the ensuing analysis.

Finaliy, we establish a uniqueness result.

Theorem 3: If $\alpha>0$ or $\pi>0$, then there is a unique BNE. If $\alpha=0$ and $\pi=0$, then all BNE yield the same allocation of consumption. When altruism is imperfect ( $\alpha$ or $\pi$ positive), equilibrium is unique. In perticular, we lose nothing at all by focusing on symmetric equilibria for the case of symmetric endowments. In a frictioniess world, there way indeed be a multiplicity of equilibria (more on this later), but all such equilibria are equivalent, so once again our analysis inyolves no loss of generality.

Throughout the following sections, we will focus on interior equilibrie (1.e., parents ake positive transfers to their children). Since we will be primarily concerned with environments that are "alnost" symmetric and frictionless, it is sufficient to assume that

$$
u^{\prime}(w)<\beta u^{\prime}(w)
$$

As a final preliminary step, we describe two types of "fiscal" policies of perticular interest. The first of these corresponds to the use of government debt. The level of debt, $\delta$, affects endowments as follows: 


$$
\begin{gathered}
-9- \\
\frac{d w_{i}}{d \delta}=1 \\
\frac{d w_{1}}{d \delta}=-1
\end{gathered}
$$

for all i. That is, the government redistributes resources from the younger generation to the older generation, presumably by deferring taxes into the future. Note that this experiment is a pure case of intergenerational redistribution, sirce all members of the same generation are affected identically.

The second type of fiscal policy considered here amounts to a pure redistributions within the perents' generation. In particular, $\tau_{i}$ represents a transfer to parent $p_{i}$, financed out of "general revenues":

$$
\frac{d W_{j}}{d \tau_{i}}= \begin{cases}1 \text { if } j=1 \\ -1 /(N-1) & \text { otherwise }\end{cases}
$$

It would also be natural to analyze a third type of policy, consisting of redistributions within the children's generation. Analytically, such policies are extremely similar to redistributions within the parent's generation, so we do not consider them explicitly. Note that, taken together, these three sets of instruments are comprehensive, in the sense that they allow the government to achieve any conceivable distribution of resources.

Throughout the rest of this paper, we focus on the extent to which fiscal instruments redistribute consumption in equilibrium. For each policy $p$ (where $p$ is either public debt, $\delta$, or an intragenerational transfer, $\left.\tau_{i}\right)$, we define a distributional index: 


$$
R^{P}=\sum_{j=1}^{N}\left(\left|\frac{d C}{d p}\right|+\left|\frac{d c_{j}}{d p}\right|\right) / 2
$$

The logic of this index is straightforwerd. If the policy $p$ ras no effect on the consumption of any individual, then $R^{p}=0$. Thus, $R^{\delta}=0$ corresponds to the Ricardian equivelence hypothesis, and $R^{\tau}{ }=0$ for ali $i$ corresponds to the Dernheim-Eagwell neutrality proposition. Note also that, in tive absence of operative intergenerational linkages, for each of the policies described above, $R^{p}=1$ (redistributing endownents leads to a one-for-one. redistribution of consumption). Thus, a value of $\mathrm{F}^{\mathrm{p}}$ between $\mathrm{O}$ and 1 telis us how closely behavior conforms to each of the polar cases.

\section{Perfect Bltruian}

We begin by considering a frictionless world, in which altruism is perfectly nonpaternalistic $(\alpha=\pi=0)$. Since such environments have received much prior attention (see Berro [1974] and Bernheim and Bagwell [1988]), this section contains no new results as such. Rather, we restate known neutrality results within the context of our current model in order to provide a "base case" with which to compare the results of subsequent sections.

Under the assumptions specified in section 2 , an interior equilibrium must satisfy

$$
u^{\prime}\left(c_{i}^{*}\right)=\beta u^{\prime}\left(c_{i}^{*}\right)
$$

$$
u^{\prime}\left(c_{i}^{*}\right)=\beta u^{\prime}\left(c_{i+1}^{*}\right),
$$


where

$$
C_{i}^{*}=W_{i}-T_{i}^{*}-t_{i}^{*}
$$

and

$$
c_{i}^{*}=w_{i}+T_{i}^{*}+t_{i-1}^{*}
$$

for all i. Given our concavity assumptions, these conditions are also sufficient to establish an equilibriur. Note that (4A), (4B), (5A), and (5B) form a system of $4 N$ equations in $4 \mathrm{~N}$ unknowns. Ordinarily, one would think that the systen would be fully determined. However, brief inspection reveals that one of the equations given in $(4 A)$ and $(4 B)$ is redundant (recall that $\left.\mathrm{C}_{\mathrm{N}+1} \equiv \mathrm{C}_{1}\right)$. Thus, the system is underdetermined.

This does not, however, reflect real indeterninancy of resource allocation. To see this, we sum (5A) and (5B) over i to obtain

$$
\sum_{i=1}^{N}\left(c_{i}^{*}+c_{i}^{*}-w_{i}-w_{i}\right)=0
$$

Note that $(4 A),(4 B)$, and (6) (omitting the redundant equation) form a system of $2 N$ equations in $2 N$ unkowns. Accordingly, it seems likely that consumption is fully determined. In fact, we have already established that there is a unique solution to this system of equations (Theorem 3).

In contrast, transfers are indeterminant. To understand this point, refer again to figure 1. Suppose that an equilibrium prevails. If every parent simply increases $T_{i}$ by $\$ 1$ and decreases $t_{i}$ by $\$ 1$, 
the allocetion of real resources remains unchanged. Thus, the new profile of transfers is also an equilibrium. Equilibrium transfers are therefore defined only up to an addive constant, with the sole restriction that all transfers must be positive.

Accordingly, we mey ignore transfers completely, and describe the equilibrium consumption profile directly through equations $(4 A),(4 B)$, and (6). Simple inspection of these equations reveais that the 3llocation of resources depends only upon total wealth,

$$
\sum_{i=1}^{N}\left(w_{i}+w_{i}\right)
$$

Changes in the distribution of wealth have no effect on the consumption of any individuel.

Several neutrality results follow immediately from this observation. We begin with Barro's [1974] well-known version of Ricardian equivalence:

$$
\text { Proposition 1: If } \alpha=\pi=0 \text {, then } R^{\delta}=0 \text {. }
$$

The proof simply consists of noting that

$$
\frac{d}{d \delta}\left(\sum_{i=1}^{N}\left(w_{i}+w_{i}\right)\right)=0,
$$

and invoking the preceding observations.

Bernheim and Bagwell [1988] have criticized Barro's analysis on two grounds. First, they argue that, in a world with intrafamily linkages, Barro's assumptions (perfect non-paternalistic altruism 
coupled with operative transfers) imply that all redistributional policies are neutral. In the current context, we obtain

$$
\text { Proposition 2: If } \alpha=\pi=0 \text {, then } R^{{ }^{T}}=0 \text { for all } i
$$

This result follows directly from the observation that

$$
\frac{d}{d \tau_{j}}\left(\sum_{i=1}^{N}\left(w_{i}+w_{i}\right)\right)=0,
$$

ana logously to Proposition 1.

Proposition 2 indicates that policies that redistribute resources between apparently unrelated members of the same generation have no effects on resource allocation. Using this result, one can also show that, in somewhat more elaborate environments, apperently distortionary taxes have no effects on behavior, and that prices are not only indeterminate, but also play no role in the resource allocation process (see Bernheim and BagweIl [1988] and Bernheim [1986]).

Bernheim and Bagwell also offered, but did not emphasize, a second criticism of the dynastic framework: as the population size increases, each individual's marginal propensity to consume out of his own wealth falls to zero. As we shall see, this observation turns out to be perticularly important in models that incorporate small amounts of friction. In the current context, we have

Proposition 3: If $\alpha=\pi=0$, then $\lim _{N \rightarrow \infty} \frac{\mathrm{dC}_{i}^{*}}{d W_{i}}=0$ for all $i$.

To establish Proposition 3, we argue as follows. By Proposition 2 (along with a similar result for children), equalizing the distribution 
of resources within generations has no effect on consumption. Thus, we can invoke Theorem 3, to conclude that the distribution of consumption is symmetric both before and after the incremental infusion of wealth. It is trivial to check that $C\left(\equiv c_{i}\right.$ for all i) and $c\left(\equiv c_{i}\right.$ for ali i) are both increasing in aggregate resources. Thus, $\mathrm{dC}_{i} / \mathrm{dW}_{i}<1 / N$, from which the result follows immediately. Empirically speaking, Proposition 2 (alone with its corollaries) and Proposition 3 are both untenable. Indeed, since these properties do not hold even as an approximation in the real. world, reality is in some critical sense not even approximately like the model described here. Accordingly, Bernheim and Bagell conclude that it is inapropriate to take the Ricardian equivalence result even as a "rule of thumb" guide to policy, wi thout first specifying the nature of the approximation in great detail. We undertake this task in subsequent sections.

\section{Joy of Giving}

In this section we analyze the case in which all parents are altruistic and, in addition, care directly about the size of the transfers they ake. Formeliy, $\alpha>0$ and $\pi=0$. Parent $i$ chooses the transfers $T_{i}$ and $t_{i}$ to satisfy

$$
\begin{aligned}
& u^{\prime}\left(C_{i}\right)=\beta u^{\prime}\left(c_{i}\right)+\alpha v^{\prime}\left(T_{i}\right) \\
& u^{\prime}\left(c_{i}\right)=\beta u^{\prime}\left(c_{i+1}\right)+\alpha v^{\prime}\left(t_{i}\right)
\end{aligned}
$$

In deciding on the optimal transfers, parent $i$ considers reducing his own consumption, $C_{i}$, by one unit. If he transfers this unit to child 
$i$, the perent's utility is increased by $\beta u^{\prime}\left(c_{i}\right)+\alpha v^{\prime}\left(T_{i}\right)$; if he transfers this unit of consumption good to child $i+1$, then the parent's utility is increaged by $\beta u^{\prime}\left(c_{i+1}\right)+\alpha v^{\prime}\left(t_{i}\right)$. The first-order conditions $(7 A)$ and $(7 B)$ show that a consumer chooses $T_{i}$ and $t_{i}$ so that the marginal utility loss from decreasing his own consumption is equal to the maginal utility gain from increasing either $T_{i}$ or $t_{i}$. To obtain compative static results for this model, one differentiates the entire system formed by equations $(7 A)$ and $(7 B)$ (for each i), along with the budget congtraints. The following result is extremely helpful for evaluating the effects of specific policy exercises.

Theorem 4: If $\pi=0, \alpha>0$, and the initial distribution of endowments is symmetric, then

$$
\begin{gathered}
\frac{d C_{j}^{*}}{d W_{k}}=\left(\lambda^{|j-k|}+\lambda^{N-|j-k|}\right)\left(1-\lambda^{N}\right)^{-1}\left(\lambda^{-1}-\lambda\right)^{-1} \frac{\alpha v^{\prime \prime}(T)}{u^{\prime \prime}(C)} \\
\qquad\left(2+\frac{\alpha v^{\prime \prime}(T)}{\beta L^{\prime \prime}(C)}\right)
\end{gathered}
$$

where $\lambda$ solves

$$
\lambda+\lambda^{-1}=2\left[1+\frac{\alpha v^{\prime \prime}(T)}{\beta u^{\prime \prime}(c)}+\frac{\alpha v^{\prime \prime}(T)}{u^{\prime \prime}(C)}\right]+\frac{\left[\alpha v^{\prime \prime}(T)\right]^{2}}{\beta u^{\prime \prime}(C) u^{\prime \prime}(c)}
$$

Since the formula for $\lambda$ is quadratic, there are, of course, two solutions. Given the nature of this formula, one root is simply the inverse of the of the other. If $\alpha>0$, then the expression on the right hand side strictly exceeds 2 , so that one solution exceeds unity, 
while the other lies between 0 and 1 . It is easy to check that the value of $\mathrm{dC}_{\mathrm{j}}^{*} / \mathrm{dW}$ does not depend upon whether one uses the larger or smaller root. For convenience, wenceforth adopt the convention that $0<\lambda<1$.

Now consider the effects of a Ricardian redistribution in which $W_{i}$ is increased by one unit and $w_{i}$ is decreased by one unit for all $i$. It is of course feasible for all $C_{i}$ and $c_{i}$ to renain unchanged in the face of this experiment. However, this invariance of consumption is not, in generel, optimal as argued below.

Suppose thet all parents meintain theis own consumption unchanged and increase $T_{i}$ and $t_{i}$ each by 1/2. In this case all $c_{i}$ will be unchanged. However, the first-order conditions (7A) and (7B) will fail to be satisfied because the increase in transfers leads to a reduction in $\alpha v^{\prime}\left(T_{i}\right)$ and $\alpha v^{\prime}\left(t_{i}\right)$. Therefore, the merginal utility of parent i's consumption, $\mathrm{I}^{\prime}\left(\mathrm{C}_{1}\right)$, would exceed the right-hand sides of $(7 \mathrm{~A})$ and $(7 \mathrm{~B})$, which represent the marginal utility associated with an additional transfer. To re-establish optimality, parent $i$ would increase his own consumption and decrease his transfers. Therefore, the Ricardian experiment increases the consumption of parents and reduces the consumption of children.

The argument that consumption would not remain unchanged in the face of a Ricardian experiment was based on the fact that increased transfers would reduce $\alpha v^{\prime}\left(T_{i}\right)$ and $\alpha v^{\prime}\left(t_{i}\right)$ and therefore violate the first-order conditions (7A) and (7E). However, if $\alpha$ is small, then this effect will be small and the impact on consumption will be minimal. 
Thus, we would expect the effect of deficits on consumption to be continuous in $\alpha$. Likewise, one would expect to obtain a similar continuity property with respect to the effect of transfer policies on consumption. Formally, we have

\section{Proposition 4: If $\pi=0$ and the initial distribution of} endowrents is symmetric, then $\lim _{\alpha \rightarrow 0} R^{\delta}=\lim _{\alpha \rightarrow 0} R^{\tau} i=0$.

Proposition 4 telis us that by taking friction to be small we can obtain both Ricardian equivalence and the stronger neutrality properties as arbitrarily good approximations. By itself, this result does not bolster the Ricardian position. However, the key point is that for $\tau_{i}$ the quality of the approximation depends upon $N$, whereas for $\delta$ it does not. Indeed, since public debt does not alter the symmetry of endowments, then by Theorem $2 R^{\delta}$ is completely independent of $N$. In contrast, $R^{\tau}$ varies with systematically with $N$.

In keeping with the intultion given in the introduction to this paper, we wish to explore the behavior of $R^{\tau_{1}}$ as $N$ becomes very large. We therefore consider in detail the effect of $\tau_{i}$ on the distribution of consumption in large economies. Note that $d C_{i} / d \tau_{i}$ is the sum of two components: (1) the direct effect of the increase in parent i's wealth, $d C_{i} / d w ;$ and (2) the effect on parent i's consumption of the reduction in parent j's wealth by $(N-1)^{-1}$ units, for all $j \neq i$. To evaluate these components in a large economy, we take the limit of the formula given in Theorem 4 (recalling that, since endowments are symetric, $C, C$, and $T$ do not depend on $N$ ): 


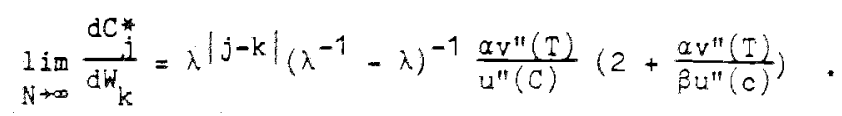

Thus, an increase in $W_{k}$ has a positive effect on $C_{j}$ but the magnitude of the effect declines gecmetrically as $|j-k|$ increases-friction dissipates the effect on more distant relatives. We depict this pattern graphically in figure 2.

Consider now the two component effects of $\tau_{i}$. For a large economy, the effect on parent $j^{\prime} s$ wealth is negligible. Indeed, it follows from Theorem 4 that even summing over all $j \neq i$, there is no effect on parent is consumption (i.e. effect (ii) above is equal to zero). Intuitively, in large econories almost all $j$ are only distantly related to $i$, so that the friction in any chain linking $j$ to $i$ almost completely dissipates the effects of changes in $j$ 's wealth. Thus, in a large economy, the effect on parent i's consumption. of the redistributive transfer $\tau_{i}$ is the same as the effect on parent i's consumption of an increase in parent i's wealth (effect (i) above). Inspection of Theorem 4 reveals that, even in a large economy, $\mathrm{dC} / \mathrm{dW}$ is positive. This follows from the fact that if parent $i$ received an additions unit of weelth and did not increase his own consumption, then he would increase his transfers $T_{i}$ and $t_{i}$, thereby increasing $c_{1}$ and $c_{i+1}$. In this case, the marginal utility of his own consumption, $U^{\prime}\left(C_{1}\right)$, would exceed the right-hand sides of the firstorder conditions (7A) and (7B). In order to satisfy the first-order conditions, perent i would increase his ow consumption. 
Finally, since the effects of wealth injections are localized, in large economies we would expect $\tau_{1}$ to redistribute consumption from the general population to the close relatives of 1 , so that in the limit $R^{i} \rightarrow 1$. We summarize these conclusions in Proposition 5.

Proposition 5: If $\pi=0, \propto>0$, and the initial distribution of endowments is symmetric, then $\lim _{N \rightarrow \infty} R^{i}=1$. Furthermore, $\lim _{N \rightarrow \infty} \frac{d C_{i}^{*}}{d \tau_{i}}=\lim _{N \rightarrow \infty} \frac{d C_{i}^{*}}{d W_{i}}>0$.

Taken together, Propositions 4 and 5 may well appear to resolve the difficulties raised by Bernheim and Bagwell. Specifically, one can obtain Ricardian equivalence to an arbitrarily good approximation by taking $\alpha$ sufficiently small... If for a given $\alpha$ the population is sufficiently large then, as in a model with no altruistic linkages, a one dollar intragenerational transfer will redistribute one dollar of consumption in equilibrium (i.e. $R^{\tau} \rightarrow 1$ ). The recipient of such a transfer will act as though he has received an injection of new wealth-that is, he will completely ignore the fact that the government acquired these resources by levying taxes on individuals to whom the recipient is operatively linked. Taking the population to be large does not, however, affect the approximate validity of Ricardian equivalence. Thus, with $\alpha$ small and $N$ large relative to $\alpha^{-1}$, deficits are approximately neutral, but intragenerational redistributions are not. Formally, we have 
Proposition 6: There exists a decreasing function $\hat{N}$ such that for any sequence $\left\langle\alpha_{k}, N_{k}\right\rangle_{k=1}^{\infty}$ with $i_{k \rightarrow \infty}\left(\alpha_{k}, N_{k}\right\rangle=(0, \infty)$ and $N_{k} \geq \hat{N}\left(\alpha_{k}\right)$ for $a 11 \quad k, \lim _{k \rightarrow \infty} R^{\delta}=0$ and $\lim _{k \rightarrow \infty} R^{\tau} i=1$. Note that one does not obtain $R^{\delta}+0$ and $R^{\tau}{ }^{i}+1$ for ail sequences $\left(\alpha_{k}, N_{k}\right) \rightarrow(0, \infty)$. W $N_{k}$ must be sufficiently large for each $a_{k}$ for the argument to work. More generally, $\left(a_{k}, N_{k}\right) \rightarrow(0, \infty)$ is consistent with any limiting value for $R^{\tau} i$, including 0 . Thus, one cannot justify Ricardian equivalence simply by arguing that friction is small and the population is large. However, the logical puzzle posed by Eernheim and Bagwell appears for the moment to be mitigated. It seems that one must turn to empirical evidence in order to determine whether the actual values of $\alpha$ and $N$ are consistent with approximate Ricardian equivalence, but inconsistent with the collateral neutrality results.

Yet this resolution is unsatisfactory. If one simulaneousiy takes a small (so that Ricardian equivalence is approximately true) and $N$ large (so that intragenerational transfers remain relevant), then in the limit each individual's consumption is necessarily unrelated to his own wealth. More precisely,

Proposition 7: Suppose $\pi=0$, and that the initial distribution of endowments is symmetric. Let $\left\langle\alpha_{k}, N_{k}\right\rangle_{k=1}^{\infty}$ be such that $\lim _{k \rightarrow \infty}\left(\alpha_{k}, N_{k}\right)=(0, \infty)$. Then 


$$
\lim _{k \rightarrow \infty}\left(\frac{d C_{i}^{*}}{d W_{i}}\right)_{k}=0
$$

Thus, by introducing friction through $a$, one cannot simultaneously resolve the difficulties raised by ?ropositions 2 and 3 of section 3 : if one takes friction to be small without letting the population get very large, then in the limit everything is neutral; if one takes friction to be smali while letting the population grow, then in the limit each individual's marginal propensity to consume out of wealth falls to zero.

Propositions 6 and 7 ma at first appear to be inconsistent. Suppose we take some sequence $\left(\alpha_{k}, N_{k}\right) \rightarrow(0, \infty)$ with $N_{k} \geq \hat{N}\left(\alpha_{k}\right)$ for each $k$. By proposition 7 , we know that in the limit consumption does not depend upon an individual's own wealth. This seems to imply that consumption depends upon aggregate wealth, from which it would follow that all redistributive policies are neutral. Quite to the contrary, proposition 6 tells us that $R^{\tau}+1$. The key to this puzzle is the fact that, in the limit, consumption is a function of local aggregates, rather than global aggregates. That is, the consumption of individual $i$ depends only upon the wealth holdings of i's "close" relatives. In the limit, $i$ has an infinite number of close realtives (even though these relatives form a negligible subset of the entire population), and so i's own wealth is irrelevant. However, a redistribution of one dollar from $i$ to $j$ (where $i$ and $j$ are only very distantly related) will transfer one dollar of consumption from $i$ and his close relatives to $j$ and his close relatives. 
In sumary, we find that one can simultaneously take friction small $(\alpha+0)$ and population large $(N \rightarrow \infty)$ such that Ricardian equivalence holds arbitrarily well, and such that redistributions have real effects (chenges in wealth only affect consumption locally). However, in doing so one necessarily produces an unterable result: each individua $1 s^{\prime}$ consumption is unrelated to his own wealth.

\section{Incomplete Information}

Now consider an economy in which a fraction $\pi$ of the parents are selfish and the remaining fraction $1-\pi$ of the parents are altuistic. Each parent knows whether he is altruistic or selfish, and knows the fraction $\pi$ of selfish parents, but does not know whether any other particular parent is selfish or altruistic. For simplicity, we assume that there is no joy of giving motive $(\alpha=0)$.

Rather than treat this case in detail, we will simply indicate its formal similarity to the joy of giving model. Specifically, if $\alpha>0$ and $\pi=0$, the utility of each parent is given by

$$
u\left(C_{i}\right)+\beta\left[u\left(y_{i}\right)+u\left(y_{i+1}\right)\right]+\alpha\left[v\left(T_{i}\right)+v\left(t_{i}\right)\right],
$$

where

$$
c_{i}=w_{i}-T_{i}-t_{i}
$$

and

$$
y_{i}=w_{i}+T_{i}+t_{i-1} \cdot
$$


Alternatively, when $\alpha=0$ and $\pi>0$, parent i's expected utility (given that he is altruistic) reduces to

$$
u\left(C_{i}\right)+\beta(1-\pi)\left[u\left(y_{i}\right)+u\left(y_{i+1}\right)\right]+\beta \pi\left[u\left(w_{1}+T_{i}\right)+u\left(w_{i+1}+t_{i}\right)\right]
$$

(recall that, with incomplete information, we interpret $T_{j}$ and $t_{j}$ as choices conditional upon $j$ being altruistic, with the understanding that $j$ trangfers nothing if he is completely selfish).

We note four differences between ( 8 ) and (9). First, in (9) the second term is multiplied by $(1-\pi)$. Clearly, this difference in scale can have no qualitative consequences, and even quantitative differences disappear as $\pi$ goes to 0. Second, in ( 9 ) the third term is multiplied by $\pi$ rather than $\alpha$. Yet both $\pi$ and $\alpha$ are measures of friction. Merely changing the index is inconsequential. Third, in

(9) $u(\cdot)$ appears in place of $v(\cdot)$. Since we never ruled out the possibility that $u(\cdot)$ and $v(\cdot)$ are identical, this too is irrelevant. Finally, in $(9) w_{i}+T_{i}$ appears in place of $T_{i}$ (likewise $w_{i+1}+t_{i}$ in place of $t_{i}$ ). Clearly, this cannot affect comparative statics for the instruments $\tau_{i}$, since $w_{j}$ in independent of $\tau_{1}$

Given the strong similarities between ( 8 ) and ( 9 ), it should not be surprising that formal analysis of the two models is virtually identical. We therefore treat these models simultaneously in Appendix A by analyzing a slightly more general formulation that subsumes both specifications. Since Appendix A gives a complete characterization of comparative statics for the general formulation, it is possible to 
obtain direct analogs of Propositions 4 through 7 for the case of $\alpha=0, \pi>0$ by mimicking the proofs in Appendix C. We leave details to the interested reader.

\section{Egelitarianiae}

Despite its apparent promise, the introduction of friction does not appear to resoive successfully all of the puzzles posed in Bernheim and Bagwell's analysis. We now turn to a less obvious alternative, which is motivated by the empirical observation that testators often choose to divide bequests equaliy among their heirs (see Menchik [1980]). This phenomenon has puzzled previous analysts, in that it appears to contradict the implications of all widely subscribed theories concerning bequest motives (see the discussion in Bernheim, Shleifer and Summers [1985]). We offer no new explanation of equal division here, but rather simply assume that altruistic parents maxime utility subject to an egalitarian constraint. Like the introduction of friction in section 4 , the constraint itself is somewhat ad hoc, but, as we shall see, its introduction generates some intruiging implications. We leave the task of justifying the equal division assumption for future work. Accordingly, we set $\alpha=\pi=0$, and modify our basic model by assuming that parent $p_{i}$ maximizes utility subject to the constraint that

$$
t_{i}=T_{i}
$$

Forma $11 y$, Theorems 1 through 3 do not apply to this case. We therefore 
provide the following result:

Theorem 5: Suppose $\alpha=\pi=0$, and that parents face egalitarian constraints. For every endowment profile $\left(w_{i}, w_{i}\right)_{i=1}^{N}$ there exists a unigue equilibrium. Furthermore, if endownents are distributed

symmetrically, then the equilibrium is symmetric, and the associated equilibrium transfer, $\mathrm{T}^{*}$, is independent of $\mathrm{N}$.

As in section 4 , it is useful to derive some preliminary comparative static results that allow us to compute the effects of various policy experiments. We therefore provide the following theorem:

Therem 6: Suppose $a=\pi=0$, and that parents face egalitarian constraints. Let initial endowments be distributed symmetrically. Then

$$
\frac{d C^{*}}{d W_{k}}= \begin{cases}-2 \lambda\left(1+\lambda^{N}\right)^{-1}\left(1-\lambda^{N}\right)^{-1}(1-\lambda)^{-1} & \text { for } j=k \\ -\left(\lambda|j-k|+\lambda^{N-|j-k|}\right)(1+\lambda)\left(1-\lambda^{N}\right)^{-1}(1-\lambda)^{-1} & \text { otherwise }\end{cases}
$$

where $\lambda$ solves

$$
\lambda+\lambda^{-1}=-2-4 \frac{u^{\prime \prime}(C)}{\beta u^{\prime \prime}(c)}
$$

Once agein, the formula for $\lambda$ is quadratic. Since the right hand side is strictly less than -2 , one solution is less than -1 , while the other lies between 0 and -1 (one is simply the reciprocal of the other). For convenience, we choose the second root (both yield the same value of $\left.\mathrm{dC}_{\mathrm{j}}^{*} / \mathrm{dW}_{\mathrm{k}}\right)$, and adopt the convention that $0>\lambda>-1$. We begin our analysis of egalitarian altruism by noting that 
Ricardian equivalence holds exactly (i.e., not approximately, as in the preceding sections). To establish this property, we need not assume that endowments are distributed symmetricaly--the result obtains even when the financial status of children differs within families.

Eroposition 8: Suppose $\alpha=\pi=0$, and that parents face egalitarian constraints. Then $R^{\delta}=0$.

It is important to qualify Proposition 8 in the following way. The previous models yielded Ricardian equivalence for approximate equivalence) for all transfers involving a parent and his children. Here, that is not the case. Policies that entail differential treatment of children within the same family may well have real effects, since the egalitarian constraint prevents parents from offsetting such redistributions.

This observation leads naturally into our next result. Just as the equal division requirement prevents parents from offsetting redistributions within the family, it precludes private individuals from offsetting more complex transfer policies. Suppose for example that the government taxes parent $p_{i}$, and distributes the procedes to $p_{i+1}$. In the absence of egalitarianism, $p_{i}$ will decrease $t_{i}$ by the amount of his incrementel tax, and $P_{i+1}$ will raise $T_{i+1}$ by his incremental subsidy. In the presence of an egalitarian constraint, these alternatives are proscribed. Instead, the actual responses of $p_{1}$ and $p_{1+1}$ will offset the policy only partially. Accordingly, one might well suspect that egalitarianism introduces 
a kind of friction, which attenuates the effects of a perturbation as one moves further from its source. In large populations, one might once again find that policies of intragenerational redistribution lead to senstble consequences. Taking limits of the formulas in theorem 6 (and recalling that, with symmetric endowments, $\lambda$ and the equilibrium allocation are independent of $N$ ), we obtain

$$
\begin{aligned}
& \frac{d C_{j}^{*}}{d W_{j}}=-2 \lambda(1-\lambda)^{-1}, \text { and } \\
& \frac{d C_{j}^{*}}{d W_{k}}=-\lambda|j-k|(1+\lambda)(1-\lambda)^{-1} \text { for } j \neq k
\end{aligned}
$$

As expected, the effect of $P_{i}$ 's wealth on $P_{j}$ 's consumption declines geometrically as $j$ becomes "distant" from $i$. However, the most strlking feature of these formulas follows from the fact that $\lambda$ is negative. Accordingly, a windfall for parent $p_{i}$ raises the consumption of $p_{j}(i \neq j)$ when $i-j$ is odd, and lowers it when $i-j$ is even (see figure 3 ).

A moment's reflection suggests that this pattern is quite natural. In response to an infusion of wealth, parent $p_{1}$ increases both his consumption and his transfers. Upon seeing that one child $\left(k_{i}, k_{i+1}\right)$ is better off, parents $p_{i-1}$ and $p_{i+1}$ choose to consume more and transfer less. As a result, the resources of children $k_{i-1}$ and $k_{i+2}$ fal1. Parents $p_{i-2}$ and $p_{i+2}$ respond by choosing to consume less, and transfer more. The pattern then repeats.

From these results it is easy to establish the relevance of 
intragenerational redistributions in large economies. In fact, parent $P_{i}$ will respond to a transfer funded from general revenues $\left(\tau_{i}\right)$ just as he would to an injection of new wealth: furthemore, the pure wealth affect does not varish as the population grows.

\section{Proposition 9: Suppose $\alpha=\pi=0$, and that parents face}

egalitarian constraints. Let initial endowiments be distributed symmetrically. Then $\lim _{N \rightarrow \infty} \frac{d C_{i}^{*}}{d \tau_{i}}=\lim _{N \rightarrow \infty} \frac{d C_{i}^{*}}{d W_{i}}>0$, and $\lim _{N \rightarrow \infty} R^{\tau}>1$.

This result has one rather peculiar implication, which is that an intragenerational transfer has a larger redistributive effect on consumption if there are egalitariar intergenerational transfers, than if there are no private transfers at all (i.e. $R^{{ }^{\top}} i>1$ ). That is, contracy to the implications of previous analyses, private transfers serve to magnify rather than dampen the redistuibutive effects of government policies.

Even so, it might appear that egalitarianism provides the ideal resolution to the paradoxes raised by Bernheim and Bagwell. After all, one obtains exact Ricardian equivalence without assuming that this source of friction is small. In contrast to previous sections, one need not pass to two limits simultaneously, thereby producing a paradoxical wealth effect.

Yet this conclusion is premature, for the imposition of egalitarianism produces a paradox of its own. Specifically, consider the welfare effects of an exogenous increase in the wealth of some consumer. Ordinarily, we would think of this occurrence as 
unambiguously desirable. Not so within the context of the current model! Indeed, roughly speaking, only one half of the population would benefit, while the other half would lose. Formally,

\section{Proposition 10: Suppose $\alpha=\pi=0$, and that parents face}

\section{egalitarian constraints. Let initial endowments be distributed}

\section{symmetrically.}

a) If $N$ is even and $j \neq 1$, then $d U_{j} / d W_{1}<0$ iff $j$ is odd.

b) If $N$ is odd and $1 \neq \min (j, N-j+2)<N / 2$, then $\mathrm{dU}_{j} / \mathrm{d} W_{1}<0$ iff $\min (j, N-j+2)$ is odd.

Thus, an exogenous increase in the wealth of any given consumer is never a Pareto improvement. The intuition for this result follows directly from our discussion of figure 3 ; if parents $j-1$ and $j+1$ consume more (and, accordingly, give less to their children), then the resources of j's family have declined, and $j$ must be worse of even after adjusting his own behavior optimally.

The reader may well feel that the implications of Proposition 10 , while surprising, are not obviously counterfactual. We do not deny this. We merely note that one cannot accept the egalitarian framework without reexaming the validity of some very basic premises, and abendoning most simple guides to welfare analysis.

\section{Closing Remarks}

In closing, it is important to emphasize that we have conducted this analysis in a way that is likely to significantly overstate the plausibility of approximately Ricardian worlds. More generally, the 
case for Ricardian equivalence is even less compelling for two reasons. First, our model spans only two generations. While it is therefore sdequate for analyzing the effects of deferring taxes to the next generation, it is unsuited for drawing inferences about the impact of longer term debt. Just as friction compounds through successive linkages between families, it will also compound as intergenerational chains lengthen. Accordingly, in a more general model, we would expect to find that relatively temporary deficits are approximetely neutral, while relatively permanent ones are not.

Second, intrafamily linkages are actually much more complicated than the network modelled here. As we extend consideration to a larger number of generations, we generate a proliferation of paths linking different members of the same generation (see Bernheim and Bagwell, section 4, for a detailed discussion). Linkages actually form a "web", rather than the circle illustrated in figure 1. As a result, the "distance" between two arbitrarily selected individuals may be quite small on average, even when the population is quite large. Suppose, for example, that we add one more generation, maintaining our assumption that every parent has two children, and every child two parents. Then, ignoring redundancies (i.e., sibling don't have the same in-laws), each grandparent is directly linked through his grandchildren to 10 other grandparents, who are in turn linked to 10 others, and so forth. This suggests that each household is connected through chains involving L or fewer links to on the order of $10^{\mathrm{L}}$ other households, rather than to only 24 households, as in the current model. Formal anelysis of 
random graphs indicates that this intuition is essentially correct (see Bollabas [1981]).

These observation suggest that, in a more realistic model, the Bernheia-Bagwell puzzles would be much more robust. If most individuals are connected through relatively few links, then it mey be very difficult to eliminate the approximate neutrality of intragenerational transfers without assuming $\alpha$ or $\pi$ very large. Similarly, each individual would in such a world have a tremendous number of "close" relatives so that, once again, the marginal propensity to consume out of own wealth might be extremely small in the absence of large friction. Overall, it is very difficult to see how one could introduce just enough friction in a model with a realistic pattern of interfamily linkages to produce approximate Ricardian equivalence wi thout also generating untenable results as in Bernheim and Bagwell. While one can, perhaps, avoid these problems by invoking an egalitarian constraint, this alternative seems very ad hoc, and in addition generates some disturbing welfare results. Consequently, the theoretical case for Ricardian equivalence remains tenuous at best. 


\section{Appendix A \\ Complete Comparative Statics for Joy of Giving and Incomplete Information iodels}

This appendix presents the comparative statics analysis of a model that nests the joy of giving model in Section 4 and the incomplete information model in Section 5. Recall that

$$
\begin{aligned}
& c_{i}=\text { consumption of adult } i \\
& c_{i}=\text { consumption of child } i \\
& w_{i}=\text { wealth of adult } i \\
& w_{i}=\text { wealth of child } i \\
& T_{i}=\text { transfer from adult } i \text { to child } i \\
& t_{i}=\text { transfer from adult } i \text { to child } i+1 .
\end{aligned}
$$

Also recall that

$$
c_{i}=w_{i}-T_{i}-t_{i}
$$

Let $y_{i}$ denote the consumption of child i if he receives transfers from adults $i$ and $i-1$,

$$
y_{i}=w_{i}+T_{i}+t_{i-1}
$$

Let

$$
Z_{i}=\phi w_{i}+T_{i}
$$

$$
z_{i}=\phi u_{i+1}+t_{i}
$$

where is a dumm variable. In particular, if $\phi=0$, then $z_{i}$ is the transfer from adult $i$ to chilc $i$ and $z_{i}$ is the transfer from 
adult $i$ to child $i+1$. Alternatively, if $\phi=1$, then $z_{i}$ is the consumption of child $i$ if he does not receive a transfer from adult $i-1 ; z_{i}$ is the consumption of child $i+1$ if he does not receive a transfer from adult $i+1$.

Now suppose that adult $i$ chooses $T_{1}$ and $t_{i}$ to maximize

$$
u\left(C_{i}\right)+u\left[u\left(y_{i}\right)+u\left(y_{i+1}\right)\right]+\left(w\left(z_{i}\right)+w\left(z_{i}\right)\right]
$$

The equations (A1 - A5) contain both the joy of giving model and the incomplete information model. To obtain the joy of giving model, set $\mu=\beta, \eta=\alpha, \phi=0$ and $w(\cdot)=v(\cdot)$. Alternatively, to obtain the private information model, set $\mu=\beta(1-\pi), \eta=\beta \pi, \phi=1$, and $w(\cdot)=u(\cdot)$

The first-order conditions are obtained by substituting (A1 - A4) into (AS) and differentiating with respect to $T_{i}$ and $t_{i}$ :

$$
\begin{aligned}
& \left(T_{i}\right):-u^{\prime}\left(c_{i}\right)+u^{\prime}\left(y_{i}\right)+\eta w^{\prime}\left(z_{i}\right)=0 \\
& \left(t_{i}\right):-u^{\prime}\left(c_{i}\right)+H^{\prime}\left(y_{i+1}\right)+\eta w^{\prime}\left(z_{i}\right)=0
\end{aligned}
$$

Now totally differentiate the first-order conditions with respect to $T_{i}, t_{i}, W_{1}$ and $w_{i}$ to obtain

(A78)

$$
\begin{aligned}
& -u^{\prime \prime}\left(c_{i}\right)\left[d w_{i}-d T_{i}-d t_{i}\right]+\mu u^{\prime \prime}\left(y_{i}\right)\left[d w_{i}+d T_{i}+d t_{i-1}\right] \\
& +\eta w^{\prime \prime}\left(z_{i}\right)\left[\phi d w_{i}+d T_{i}\right]=0 \\
& -u^{\prime \prime}\left(c_{i}\right)\left[d W_{i}-d T_{i}-d t_{i}\right]+\mu u^{\prime \prime}\left(y_{i+1}\right)\left[d w_{i+1}+d T_{i+1}+d t_{i}\right] \\
& +\eta w^{\prime \prime}\left(z_{i}\right)\left[\phi d w_{i+1}+d t_{i}\right]=0
\end{aligned}
$$


We assume that initially $W_{i}=W$ and $w_{i}=$ for all $i$, and we restrict our attention to symmetric equilibria. Let

$$
\begin{aligned}
& a \equiv u^{\prime \prime}\left(c_{i}\right)+H u^{\prime \prime}\left(y_{i}\right)+\eta w^{\prime \prime}\left(z_{i}\right)<0 \\
& b \equiv u^{\prime \prime}\left(c_{i}\right)<0 \\
& e \equiv \mu u^{\prime \prime}\left(y_{i}\right)<0 \\
& f \equiv \eta W\left(z_{i}\right)<0
\end{aligned}
$$

and observe that $a:=b+e+f<0$. Using the definitions of $a, b, e$ and $f$ we can rewrite $(A 7 a, b)$ as

(A\&a)

$$
a d T_{i}+b d t_{i}+e d t_{i-1}=b d w_{i}-(e+f \Phi) d w_{i}
$$

$$
b d T_{i}+a d t_{i}+e d T_{i+1}=b d W_{i}-(e+f \phi) d w_{i+1}
$$

Let $x_{i}$ be $a 2 \times 1$ colum vector such that $x_{i}^{\prime}=\left[d T_{i}, d t_{i}\right]$. The linear difference equation system in ( $A B)$ can be written as

(A.9) $\left[\begin{array}{ll}a & b \\ e & 0\end{array}\right]\left[x_{i}\right]=\left[\begin{array}{cc}0 & -e \\ -b & -a\end{array}\right]\left[x_{i-1}\right]+\left[\begin{array}{cc}b d w_{i}-(e+f \phi) d w_{i} \\ b d w_{i-1}-(e+f \phi) d w_{i}\end{array}\right]$

Now observe that

(A10)

$$
\left[\begin{array}{ll}
a & b \\
e & 0
\end{array}\right]^{-1}=\left[\begin{array}{cc}
0 & \frac{1}{e} \\
\frac{1}{b} & \frac{-e}{b e}
\end{array}\right]
$$


and then pre-multiply both sides of (A9) by the matrix on the right-hand sice of (A10) to obtain

$$
x_{j}=M x_{j-1}+g_{j}
$$

where

$$
M=\left[\begin{array}{cc}
-\frac{b}{e} & -\frac{a}{e} \\
\frac{a}{e} & -\frac{e}{b}+\frac{a^{2}}{b e}
\end{array}\right]
$$

and

$$
g_{j}=\left[\begin{array}{l}
\frac{b}{e} d w_{j-1}-\left(1+\frac{f \phi}{e}\right) d w_{j} \\
d w_{j}-\frac{a}{e} d w_{j-1}+\left(\frac{a}{e}-1\right)\left(\frac{e+f \phi}{b}\right) d w_{j}
\end{array}\right]
$$

The behavior of $x_{j}=\left[d T_{j}, d t_{j}\right]$ is governed by the inear difference equation in (A11) and the boundary condition that

$$
x_{1}=M x_{N}+g_{1}
$$

The boundary condition in (A12) exploits the fact that the $N$ adults are located around a circle and adult is formally the same as adult $\mathbf{N}+1$

For the purposes of our analysis, it is sufficient to allow $b_{1}$ and $g_{2}$ to be nonzero and to cestrict $g_{j}=0$ for $j=3,4,5, \ldots, N$. In this case, it follows from (A11) and (A12) that

$$
x_{2}=M^{2} x_{N}+M g_{1}+g_{2}
$$


and

$$
x_{N}=M^{N-2} x_{2}
$$

Substituting (A14) into (A13) yields an expression for $x_{2}$ in terms of the exogenous changes $B_{1}$ and $g_{2}$

$$
x_{2}=\left(I-M^{N}\right)^{-1}\left[M g_{1}+g_{2}\right]
$$

Using the boundary condition in (A12), the expression for $x_{2}$ in (A.15) and the fact that $x_{j}=M^{j-2} x_{2}$ for $j=2, \ldots, N$ we have a complete solution for $x_{1}, \ldots, x_{N}$.

(A16a)

$$
x_{1}=M^{N-1}\left(I-M^{N}\right)^{-1}\left[M g_{1}+g_{2}\right]+g_{1}
$$

(A16b)

$$
x_{j}=M^{j-2}\left(I-M^{N}\right)^{-1}\left[M g_{1}+g_{2}\right] ; j=2, \ldots, N
$$

Let $\lambda_{1} \leqslant \lambda_{2}$ be the two characteristic roots of the matrix M. Observe thet

$$
\begin{aligned}
& \lambda_{1}+\lambda_{2}=\operatorname{tr} M=\frac{a^{2}-b^{2}-e^{2}}{b e}>0 \\
& \lambda_{1} \cdot \lambda_{2}=\operatorname{det} M=1
\end{aligned}
$$

It follows from (A17b) that $\lambda_{1}$ and $\lambda_{2}$ are reciprocals of each other. Let $\lambda$ denote the smaller root $\lambda_{1}$. It follows from (A17a) that both roots, $\lambda$ and $\lambda^{-1}$, are positive.

It can be directly verified that the matrix $M$ can be written as 
$-37-$

where

$(A 18 b)$

$$
\begin{aligned}
& P=\left[\begin{array}{cc}
1 & 1 \\
-\frac{1}{a}(b+e \lambda) & -\frac{1}{a}\left(b+e \lambda^{-1}\right)
\end{array}\right] \\
& A=\left[\begin{array}{cc}
\lambda & 0 \\
0 & \lambda^{-1}
\end{array}\right]
\end{aligned}
$$

(A18c)

and

$$
\text { (A18d) } \quad \mathrm{B}^{-1}=\frac{1}{e\left(\lambda-\lambda^{-1}\right)}\left[\begin{array}{cc}
-\left(b+e \lambda^{-1}\right) & -a \\
(b+e \lambda) & a
\end{array}\right]
$$

Now observe that

(A19)

$$
M^{j-2}\left(I-M^{N}\right)^{-1}=P A^{j-2}\left(I-A^{N}\right)^{-1} P^{-1}
$$

Substituting (A18b) and (A18c) into (A19) yields

(A20) $M^{j-2}\left(I-M^{N}\right)^{-1}=\frac{1}{1-\lambda^{N}}\left[\begin{array}{cc}\lambda^{j-2} & -\lambda^{N-j+2} \\ -\frac{1}{a}(b+e \lambda) \lambda^{j-2} & \frac{1}{a}\left(b+e^{-1}\right) \lambda^{N-(j-2)}\end{array}\right] P^{-1}$

We are now prepared to analyze two comparative statics

exercises. First, we examine the effects of an increase in the wealth

of parent 1. In particular, we let $\mathrm{dW}_{1}>0$ and

$d w_{2}=\cdots \cdot=d w_{n}=d w_{1}=\cdots \cdot=d w_{N}=0$. In this case we have

(A21a)

$$
g_{1}=\left[\begin{array}{l}
0 \\
1
\end{array}\right] \mathrm{dW}_{1}
$$




$$
g_{2}=\left[\begin{array}{c}
\frac{b}{e} \\
-\frac{a}{e}
\end{array}\right] d w_{1}^{-38-}
$$

(A210)

and

$$
M g_{1}+g_{2}=\left[\begin{array}{c}
\frac{b-a}{e} \\
\frac{a^{2}-e^{2}-a b}{b e}
\end{array}\right] d w_{1}
$$

Using (A17a) we can rewrite (A22) as

(A23)

$$
M g_{1}+g_{2}=\left[\begin{array}{l}
\frac{b-a}{e} \\
\frac{b-a}{e}+\lambda+\lambda^{-1}
\end{array}\right] d w_{1}
$$

Now observe that

(A24) $\mathrm{P}^{-1}\left[\operatorname{lgg}_{1}+g_{2}\right]=\frac{1}{e\left(\lambda-\lambda^{-1}\right)}\left[\begin{array}{c}-(b+a) \frac{b-a}{e}-(b-a) \lambda^{-1}+a\left(\lambda+\lambda^{-1}\right) \\ (b+a) \frac{b-a}{e}+(b-a) \lambda+a\left(\lambda+\lambda^{-1}\right)\end{array}\right] d w_{1}$

Observe from (A17a) that

$$
(b+a)(b-a) / e=-b\left(\lambda+\lambda^{-1}\right)-e
$$

Substituting (A25) into (A24) yields

(A26)

$$
\mathrm{P}^{-1}\left[M g_{1}+g_{2}\right]=\frac{1}{\lambda-\lambda^{-1}}\left[\begin{array}{c}
\frac{b-a}{e} \lambda+1 \\
-\frac{b-a}{e} \lambda^{-1}-1
\end{array}\right] d \omega_{1}
$$


Pre-multiply (A26) by (A20) and use (A15b) to obtain

(A27)

$$
x_{j}=\left(1-\lambda^{N}\right)^{-1}\left(\lambda-\lambda^{-1}\right)^{-1}\left[\begin{array}{c}
\lambda^{j-2}+\lambda^{N-j+2}+\frac{b-a}{e}\left(\lambda^{j-1}+\lambda^{N-j+1}\right) \\
-\frac{1}{a}\left[(b+e \lambda)\left(1+\frac{b-a}{e} \lambda\right) \lambda^{j-2}\right. \\
\left.+\left(b+e \lambda^{-1}\right)\left(1+\frac{b-a}{e} \lambda^{-1}\right) \lambda^{N-j+2}\right]
\end{array}\right] d w_{1}
$$

To simplify (A27) recall that the roots $\lambda_{1}=\lambda$ and $\lambda_{2}=\lambda^{-1}$ satisfy the characterist1c equation $k_{i}^{2}-\lambda_{i}$ tr $M+$ det $k=0$ which can be written as

$$
b e k_{i}^{2}=\left(a^{2}-b^{2}-e^{2}\right) \lambda_{i}-b e
$$

Now observe that

$(A 29)\left(b+e \lambda_{i}\right)\left(1+\frac{b-a}{e} \lambda_{i}\right)=\frac{1}{e}\left\{(b e-a e) \lambda_{i}^{2}+\left(e^{2}+b^{2}-a b\right) \lambda_{i}+b e\right\}$

Substituting (A28) into the right-hand side of (A29) yields

$$
(A 30)\left(b+e \lambda_{i}\right)\left(1+\frac{D-a}{e} \lambda_{i}\right)=-a \lambda_{i}\left[\frac{b-a}{e}+\lambda_{i}\right], \quad i=1,2
$$

Substituting (A30) into (A27) yields

$$
x_{j}=\left(1-\lambda^{N}\right)^{-1}\left(\lambda-\lambda^{-1}\right)^{-1}\left[\begin{array}{c}
\lambda^{j-2}+\lambda^{N-j+2}+\frac{b-a}{e}\left(\lambda^{j-1}+\lambda^{N-j+1}\right) \\
\lambda^{j}+\lambda^{N-j}+\frac{b-a}{e}\left(\lambda^{j-1}+\lambda^{N-j+1}\right) \\
1=2, \ldots, N
\end{array}\right] \text { dw }
$$


To calculate $x_{1}$, observe from $(A 12)$ and $(A 14)$ that $x_{1}=M^{N-1} x_{2}+B_{1}$. Eorma1ly, $x_{1}$ can be written as $x_{N+1}+g_{1}$ where $g_{1}^{\prime}=\left(0, d W_{1}\right)$. Therefore

$$
x_{1}=\left(1-\lambda^{N}\right)^{-1}\left(\lambda-\lambda^{-1}\right)^{-1}\left[\begin{array}{l}
\lambda+\lambda^{N-1}+\frac{b-a}{e}\left(\lambda^{N}+1\right) \\
\lambda+\lambda^{N-1}+\frac{b-a}{e}\left(\lambda^{N}+1\right)
\end{array}\right] d W_{1}
$$

Now we consider the alternative exercise of increasing the wealth of child 1 by $d w{ }_{1}>0$. In this case

$$
g_{1}=\left(1+\frac{f \phi}{e}\right)\left[\begin{array}{c}
-1 \\
\frac{a-e}{b}
\end{array}\right] d_{1}
$$

and $g_{2}=\cdots \cdot=g_{\mathrm{N}}=0$.

In this case, $x_{j}=M^{j-2}\left(I-M^{N}\right)^{-1} M g_{1}$ which can be written as

$$
x_{j}=P \Lambda^{j-2}\left(I-\Lambda^{N}\right)^{-1} P^{-1} P \Lambda P^{-1} g_{1} \quad j=2, \ldots, N
$$

Equation (A34) can be rearranged to yield

$$
x_{j}=P \Lambda^{j-1}\left(I-\Lambda^{N}\right)^{-1} P^{-1} g_{1} \quad j=2, \ldots, N
$$

It follows from (A18d) and (A33) that

(A36)

$$
\mathrm{P}^{-1} g_{1}=\frac{1+\frac{f \Phi}{e}}{e\left(\lambda-\lambda^{-1}\right)}\left[\begin{array}{c}
b+e^{\lambda^{-1}}+\frac{-a^{2}+a e}{b} \\
-(b+e \lambda)+\frac{a^{2}-a e}{b}
\end{array}\right] d w_{1}
$$


Now observe that $e \lambda_{1}+b+\frac{-a^{2}+a e}{b}=e \lambda_{1}-\frac{a^{2}-b^{2}-a e}{b}$ so that in light of (A17a);

(A37) $e \lambda_{i}+b+\frac{-a^{2}+a e}{b}=e\left\{\lambda_{i}-\left(\lambda_{i}+\lambda_{j}\right)-\frac{e^{2}-a e}{b e} \quad i \neq j\right.$.

Simplifying (A37) yields

$$
e \lambda_{i}+b-\frac{a^{2}-a e}{b}=e\left(-\lambda_{j}+\frac{a-e}{b}\right)
$$

Recalling that $\lambda_{1}=\lambda$ and $\lambda_{2}=\lambda^{-1}$, we can use (A38) to rewrite (A36) as

$$
\mathrm{P}^{-1} g_{4}=\frac{1+\frac{f \phi}{e}}{\lambda-\lambda^{-1}}\left[\begin{array}{c}
\frac{a-e}{b}-\lambda \\
-\frac{a-e}{b}+\lambda^{-1}
\end{array}\right] d w_{1}
$$

Now use $(A 19),(A 20),(A 35)$ and (A39) to obtain

(A40)

$$
x_{j}=\left(1+\frac{f \Phi}{e}\right)\left(1-\lambda^{N}\right)^{-1}\left(\lambda-\lambda^{-1}\right)^{-1}\left[\begin{array}{r}
-\left(\lambda^{j}+\lambda^{N-j}\right)+\frac{a-e}{b}\left(\lambda^{j-1}+\lambda^{N-j+1}\right) \\
-\frac{1}{a}(b+e \lambda)\left[\frac{a-e}{b}-\lambda\right] \lambda^{j-1} \\
+\left(b+e \lambda^{-1}\right)\left[\frac{a-e}{b}-\lambda^{-1}\right] \lambda^{N-j+1}
\end{array}\right]
$$

To simplify (A4O) observe that

(A49) $\left(b+e \lambda_{i}\right)\left(\frac{a-e}{b}-\lambda_{i}\right)=\frac{1}{b}\left\{a b-b e+\left(a e-e^{2}-b^{2}\right) \lambda_{i}-b e \lambda_{i}^{2}\right\}$

Substituting (A28) into (A41) yields

(A42)

$$
\left(b+e \lambda_{i}\right)\left(\frac{a-e}{b}-\lambda_{i}\right)=a\left(1+\frac{e-a}{b} \lambda_{i}\right)
$$


Substituting (A42) into (A4O) yields (A43)

$$
x_{j}=\left(1+\frac{f+}{e}\right)\left(1-\lambda^{N}\right)^{-1}\left(\lambda-\lambda^{-1}\right)^{-1}\left[\begin{array}{l}
-\left(\lambda^{j}+\lambda^{N-j}\right)+\frac{a-e}{b}\left(\lambda^{j-1}+\lambda^{N-j+1}\right) \\
-\left(\lambda^{j-1}+\lambda^{N-j+1}\right)+\frac{a-e}{b}\left(\lambda^{j}+\lambda^{j-j}\right)
\end{array}\right] d w_{1}^{k j-j, N .}
$$

To calculate $x_{1}$, note that formally $x_{1}=x_{N+1}+8_{1}$. Using (A43) and (A33) we obtain

$$
x_{1}=\left(1+\frac{f \Phi}{e}\right)\left(1-\lambda^{N}\right)^{-1}\left(\lambda-\lambda^{-1},-1\left[\begin{array}{l}
-\left(\lambda+\lambda^{N-1}\right)+\frac{a-e}{b}\left(1+\lambda^{N}\right) \\
-\left(1+\lambda^{N}\right)+\frac{a-e}{b}\left(\lambda+\lambda^{N-1}\right)
\end{array}\right] d w_{1}\right.
$$

\section{Appendix B}

\section{Egalitarianism}

This appendix presents the comparative statics analysis of the economy in which all parents divide their estates equally among their children. Recali that

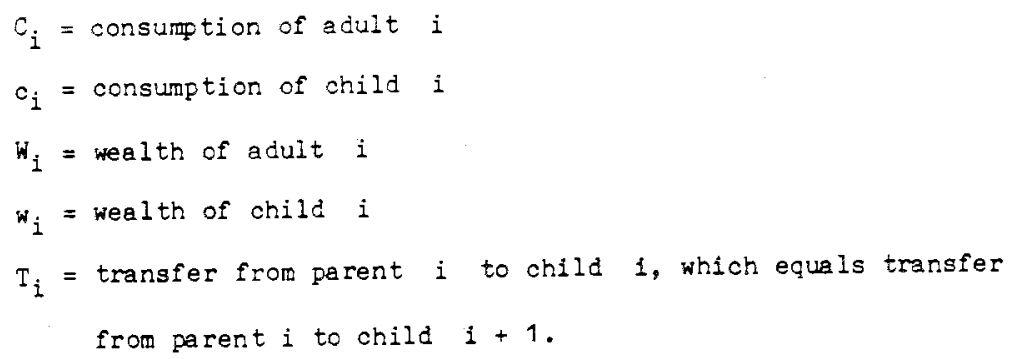

Observe that

$$
c_{i}=W_{i}-2 T_{i}
$$


and

$$
c_{i}=w_{i}+T_{i}+T_{i-1}
$$

Parent $i$ chooses $T_{i}$ to maximize

$$
u\left(c_{i}\right)+\beta\left[u\left(c_{i}\right)+u\left(c_{i+1}\right)\right]
$$

and the first-order condition for this maximization problem is

$$
-2 u^{\prime}\left(c_{i}\right)+\beta u^{\prime}\left(c_{i}\right)+\beta u !\left(c_{i+1}\right)=0
$$

Totally differentiating this first-crder condition with respect to $T_{1}$ $w_{i}$ and $w_{i}$ yields

$$
\begin{aligned}
-2 u^{\prime \prime}\left(c_{i}\right)\left[d w_{i}-2 d T_{i}\right] & +\beta u^{\prime \prime}\left(c_{i}\right)\left[d w_{1}+d T_{i}+d T_{i-1}\right] \\
& +\beta u^{\prime \prime}\left(c_{i+1}\right)\left[d w_{i+1}+d T_{i}+d T_{i+1}\right]
\end{aligned}
$$

We assume that initially $w_{i}=w$ and $w_{i}=w$ for all $i$, and we restrict our attention to symmetric equilibria. Let

$$
\begin{aligned}
& a \equiv 4 u^{\prime \prime}\left(c_{i}\right)+2 \beta u^{\prime \prime}\left(c_{i}\right)<0 \\
& b \equiv 2 u^{\prime \prime}\left(c_{i}\right)<0 \\
& e \equiv \beta u^{\prime \prime}\left(c_{i}\right)<0
\end{aligned}
$$

and observe that $a=2(b+e)$. Using the definitions of $a, b$, and $e$, we can write (B5) as

$$
e d T_{i-1}+a d T_{i}+e d T_{i+1}=b d w_{i}-e d w_{i}-e d w_{i+1}
$$


The second-order linear difference equation in (B6) can be written In companion form by defining the $2 \times 1$ column vector $x_{i}$ as $x_{i}=\left[d T_{i}, d T_{i-1}\right]$ '. Therefore,

$$
x_{i}=M x_{i-1}+h_{i} \quad, \quad i=2, \ldots, N
$$

where

$$
M=\left[\begin{array}{cc}
-\frac{a}{e} & -1 \\
1 & 0
\end{array}\right]
$$

and

$$
h_{i}=\left[\begin{array}{c}
\frac{b}{e} d w_{i-1}-\left(d w_{i-1}+d w_{i}\right) \\
0
\end{array}\right]
$$

The behavior of $x_{1}$ is governed by (B7) and the boundary condition

$$
x_{1}=M x_{N}+h_{1}
$$

The boundary condition in (Be) reflects the fact that formally adult 1 may be represented as adult $N+1$.

For the purposes of our analysis, it is sufficient to allow $h_{1}$ and $h_{2}$ to be nonzero and to restrict $h_{i}=0$ for $i=3,4,5, \ldots, N$. In this case, it follows from (B7) and (B8) that

$$
\begin{gathered}
x_{N}=M^{N-2} x_{2} \\
x_{2}=M^{2} x_{N}+M h_{1}+n_{2}
\end{gathered}
$$


Using the fact thet $x_{j}=M^{j-2} x_{2}$ for $j=2, \ldots, N$, we can use (58-810) to obtain a complete solution for $x_{1}, \ldots, x_{N}$

(B11a)

$$
\begin{aligned}
& x_{1}=M^{k-1}\left(I-M^{N}\right)^{-4}\left[M h_{1}+h_{2}\right]+h_{1} \\
& x_{i}=M^{i-2}\left(I-M^{N}\right)^{-\{}\left[M_{1}+h_{2}\right] \quad i=2, \cdots \cdots N .
\end{aligned}
$$

Let $\lambda_{1}>\lambda_{2}$ be the two characteristic roots of the matrix M.

Observe that

$$
\begin{aligned}
& \lambda_{1}+\lambda_{2}=\operatorname{tr} M=-\frac{a}{e}<-2 \\
& \lambda_{1} \cdot \lambda_{2}=\operatorname{det} M=1
\end{aligned}
$$

It follows from (B12b) that the roots are reciprocals of each other. Let $\lambda$ be the larger root $\lambda_{1}$; therefore $\lambda_{2}=\lambda^{-1}$. It follows from (B12a) that $x^{-1}<-1<\lambda<0$.

It can be directly verified that the matrix $M$ can be written as

$$
\mathrm{M}=\mathrm{PAP}^{-1}
$$

\section{where}

(B13b)

$$
P=\left[\begin{array}{ll}
1 & 1 \\
\lambda^{-1} & \lambda
\end{array}\right]
$$

$(B 13 c)$

$$
\Lambda=\left[\begin{array}{ll}
\lambda & 0 \\
0 & \lambda^{-1}
\end{array}\right]
$$


(B13d)

$$
P^{-1}=\frac{1}{\lambda-\lambda^{-1}}\left[\begin{array}{cc}
\lambda & -1 \\
-\lambda^{-1} & 1
\end{array}\right]
$$

Now observe that

$$
M^{i-2}\left(I-M^{N}\right)^{-1}=P \Lambda^{i-2}\left(I-N^{N}\right)^{-1} \mathrm{P}^{-1}
$$

Substituting ( $313 b, c, d)$ into (B14) and performing the matrix multiplication yields

$$
M^{i-2}\left(I-M^{N}\right)^{-1}=\left(1-\lambda^{N}\right)^{-1}\left(\lambda-\lambda^{-1}\right)^{-1}\left[\begin{array}{ll}
\lambda^{i-1}+\lambda^{N-i+1} & -\left(\lambda^{i-2}+\lambda^{N-i+2}\right) \\
\lambda^{i-2}+\lambda^{N-i+2} & -\left(\lambda^{i-3}+\lambda^{N-i+3}\right)
\end{array}\right]
$$

We are now prepared to analyze two comparative statics exercises.

First, we examine the effects of an increase in the wealth of adult 1. In particular, let $d w_{1}>0$ and $d w_{2}=\ldots=d w_{N}=d w_{1}=\ldots d w_{N}=0$. In this case $h_{1}=\left[\begin{array}{l}0 \\ 0\end{array}\right]$ and $h_{2}=\left[\begin{array}{c}(b / e) d w_{1} \\ 0\end{array}\right]$. It follows from (B11b) and (B15) thet

(B16)

$$
x_{i}=\left(1-\lambda^{N}\right)^{-1}\left(\lambda-\lambda^{-1}\right)^{-1}\left[\begin{array}{c}
\frac{b}{e}\left(\lambda^{i-1}+\lambda^{N-i+1}\right) \\
\frac{b}{e}\left(\lambda^{i-2}+\lambda^{N-i+2}\right)
\end{array}\right] d w_{1} i=2, \ldots, N
$$

Therefore,

$$
\frac{d T_{i}}{d W_{1}}=\frac{b}{e}\left(1-\lambda^{N}\right)^{-1}\left(\lambda-\lambda^{-1}\right)^{-1}\left[\lambda^{i-1}+\lambda^{N-i+1}\right]
$$




$$
\begin{aligned}
& -47- \\
& i=1, \ldots, N .
\end{aligned}
$$

Now consider the alternative exercise of increasing the wealth of child 1. In particular, let dw $>0$ and $d w_{2}=\cdots=d w_{N}=d w_{1}=$ $\ldots=d w_{N}=0$. In this case, $h_{1}=\left[\begin{array}{ll}-1 & 0\end{array}\right] \cdot d w_{1}$ and $h_{2}=\left[\begin{array}{ll}-1 & 0\end{array}\right] \cdot$ dw, so that

(B18)

$$
M h_{1}+h_{2}=\left[\begin{array}{c}
\frac{a-e}{e} \\
-1
\end{array}\right] d w_{1}
$$

It follows from $(\mathrm{E} 11 \mathrm{~b}),(\mathrm{B} 15)$ and $(\mathrm{B} 18)$ that

(B19)

$$
\begin{gathered}
x_{i}=\left(1-\lambda^{1}\right)^{-1}\left(\lambda-\lambda^{-1}\right)^{-1}\left[\begin{array}{l}
\frac{a-e}{e}\left(\lambda^{i-1}+\lambda^{N-i+1}\right)+\left(\lambda^{i-2}+\lambda^{N-i+2}\right) \\
\frac{a-e}{e}\left(\lambda^{i-2}+\lambda^{N-i+2}\right)+\left(\lambda^{i-3}+\lambda^{N-i+3}\right)
\end{array}\right] \\
i=2, \ldots, N
\end{gathered}
$$

Therefore,

(B20)

$$
\begin{gathered}
\frac{d T_{1}}{d w_{1}}=\frac{\frac{a-e}{e}\left(\lambda^{i-1}+\lambda^{N-i+1}\right)+\left(\lambda^{i-2}+\lambda^{N-i+2}\right)}{\left(1-\lambda^{N}\right)\left(\lambda-\lambda^{-1}\right)} \\
i=1, \ldots, N .
\end{gathered}
$$




\section{Appendix $\mathrm{C}$}

Theorem 1: Let $S_{i}=\left\{(T, t) \mid T+t \leqq W_{i}\right.$ and $\left.I, t \geq 0\right\} \cdot S_{i}$ is $p_{i}$ 's strategy space; let $s_{i}$ derote an element of $S_{i}$. Note that $S_{i}$ is compact and convex. Further, $p_{j}^{\prime}$ 's utility is by assumption continuous in $s=\left(s_{\uparrow}, \ldots, s_{N}\right)$, and (it is easy to verify) quasi-concave in $s_{j}$. Thus, by Debreu's [1952] Sccial Equilibrium Existence Theorem, there exists a profile of strategies $\left(s_{1}^{*}, \ldots, s_{N}^{*}\right)$ which satisfies our definition of equilibrium.

Q.E.D.

Theorem 2: In a symmetric equilibrium with transfer level $T^{*}$, $\left(T^{*}, T^{*}\right)$ must satisfy

$$
\begin{aligned}
\max _{T, t} u(w-T-t) & +\beta\left\{(1-\pi)\left[u\left(w+T+T^{*}\right)+u\left(w+t+T^{*}\right)\right]\right. \\
& +\pi[u(w+T)+u(w+t)]\}+\alpha[v(T)+v(t)]
\end{aligned}
$$

subject to $T \geqslant 0, t \geq 0$, and $T+t \leq W$. Ey concavity of $u$ and $v$, we know that the solution always entails $T=t$, so we simply require that $\mathrm{T}^{*}$ solves

$$
\max _{T} u(w-2 T)+2 \beta\left\{(1-\pi) u\left(w+T+T^{*}\right)+\pi u(w+T)\right\}+2 \alpha v(T)
$$

subject to $0 \leqq T \leqq W / 2$. Let $\gamma:[0, W / 2] \rightarrow[0, W / 2]$ be defined as

$$
\gamma(\hat{T})=\arg \max _{\substack{0 \\=} \leq W / 2} u(W-2 T)+2 \beta[(1-\pi) u(W+T+\hat{T})+\pi u(w+T)]+2 \alpha v(T)
$$

Since this objective function is continuous and strictly concave, $\gamma$ is a continuous function. By the intermediate value theorem, there 
exists $I^{*}$ such that $I^{*}=\gamma\left(T^{*}\right)$, as required. Einally, note that the equilibrium condition is independent of $\mathrm{N}$, so $\mathrm{T}^{*}$ remains a symmetric equilibrium independent of $N$.

Theorem 3: For any ENE $\left(T_{i}, t_{i}\right)_{i=1}^{N}$, let

$$
\begin{aligned}
& c_{i}^{0}=w_{i}-T_{i}-t_{i}, \\
& c_{i}^{0}=w_{i}+T_{i}+t_{i-1} .
\end{aligned}
$$

That is, $C_{i}^{0}$ is $p_{1}{ }^{\prime}$ s consumption contingent upon $P_{i}$ being altruistic, and $c_{i}^{0}$ is $k_{i}^{\prime} s$ consumption contingent upon $p_{i}$ and $p_{i-1}$ being altruistic. We will first establish that $\left(c_{i}^{0}, c_{i}^{0}\right)_{i=1}^{N}$ must be identical in aII BNE.

Suppose this claim is false. Then there zre two BNE which give rise to distinct profiles $\left(\mathrm{C}_{i}^{O}, \mathrm{C}_{i}^{O}\right)_{i=1}^{N}$ and $\left(\hat{\mathrm{C}}_{i}^{\mathrm{O}}, \mathrm{c}_{i}^{O}\right)_{i=1}^{N}$ wi thout loss of generality, we may suppose that ei her $\bar{C}_{j}^{0}>\hat{C}_{j}^{0}$ or $\hat{c}_{j}^{0}>\hat{c}_{j}^{0}$ for some $j$.

Take first the case of $\bar{C}_{j}^{0}>\hat{C}_{j}^{0}$. Through $P_{j}^{\prime}$ s budget constraint, we see that either $\overline{\mathrm{T}}_{j}<\hat{T}_{j}$ or $\bar{t}_{j}<\hat{t}_{j}$ wi thout loss of generality, we assume $\bar{t}_{j}<\hat{t}_{j}$

Now we use induction. Suppose that for some $i \geq 0, \bar{c}_{j+i}^{0}>\hat{c}_{j+i}^{0}$, and $\bar{t}_{j+i}<\hat{t}_{j+i}$. Since it must then be the case that $\hat{t}_{j+i}>0$, we have

$$
u^{\prime}\left(\hat{c}_{j+i}^{0}\right) \leq \beta(1-\pi) u^{\prime}\left(\hat{c}_{j+i+1}^{0}\right)+\beta \pi u^{\prime}\left(w_{j+i+1}+\hat{t}_{j+i}\right)+\alpha v^{\prime}\left(\hat{t}_{j+i}\right)
$$

(inequality may occur if $\hat{c}_{j+i}^{0}=0$ ). Now we argue that $\hat{c}_{j+i+1}^{0}>\hat{c}_{j+i+1}^{0}$. 
For suppose not. Using strict concavity of $u$ and $y$, along with $\bar{C}_{j+i}^{0}>\hat{C}_{j+i}^{o}$ and $\bar{t}_{j+i}<\hat{t}_{j+i}$, we would have

$$
u^{\prime}\left(\bar{c}_{j+i}^{0}\right)<\beta(1-\pi) u^{\prime}\left(\bar{c}_{j+i+1}^{0}\right)+\beta \pi u^{\prime}\left(w+i+1+\bar{t}_{j+i}\right)+\alpha v^{\prime}\left(\bar{t}_{j+i}\right)
$$

But this implies that $P_{j+i}$ could increase his utility by transfering more to $k_{j+i+1}$, which is a contradiction.

$$
\text { Next, since } \bar{t}_{j+i}\left\langle\hat{t}_{j+i} \text { and } \bar{c}_{j+i+1}^{0}\right\rangle \hat{c}_{j+i+1}^{0} \text {, then, from }
$$

$k_{j+i+1}{ }^{\prime}$ s budget constraint, we must have $\bar{I}_{j+j+1}>\hat{T}_{j+j+1}$. Since $i t$ must then be the case that $\overline{\mathrm{T}}_{j+i+1}>0$, we have

$$
u^{\prime}\left(\bar{c}_{j+i+1}^{0}\right) \leq \beta(1-\pi) u^{\prime}\left(\bar{c}_{j+i+1}^{0}\right)+\beta \pi u^{\prime}\left(w_{j+j+1}+\bar{I}_{j+i+1}\right)+\alpha v^{\prime}\left(\bar{I}_{j+j+1}\right)
$$

Now we argue that $\bar{c}_{j+i+1}^{0}>\hat{c}_{j+i+1}^{0}$. For suppose not. Using strict concavity of $u$ and $v$, along with $\bar{c}_{j+i+1}^{0}>\hat{0}_{j+i+1}^{0}$ and $\overline{\bar{T}}_{j+i+1}>\hat{i}_{j+i+1}$, we would have

$$
u^{\prime}\left(\hat{C}_{j+i+1}^{0}\right)<\theta(\hat{i}-\pi) u^{\prime}\left(\hat{c}_{j+i+1}^{0}\right)+\beta \pi u^{\prime}\left(w_{j+i+1}+\hat{T}_{j+i+1}\right)+\alpha v^{\prime}\left(\hat{T}_{j+i+1}\right)
$$

But this implies $P_{j+i+1}$ could increase his utility by transferring more to $k_{j+i+1}$; which is a contradiction.

Finally, if $\overline{\mathrm{T}}_{j+i+1}>\hat{\mathrm{T}}_{j+i+1}$ and $\overline{\mathrm{C}}_{j+i+1}^{0}>\hat{\mathrm{C}}_{j+i+1}^{0}$, then by $p_{j+i+1}{ }^{t} \mathrm{~s}$ budget constraint, $\bar{t}_{j+i+1}<\hat{t}_{j+i+1}$. This completes the induction step. Note that induction implies $\bar{C}_{i}^{0}>\hat{C}_{i}^{0}$ and $\bar{c}_{i}^{0}>\hat{c}_{i}^{0}$ for all $i$. This violates the aggregate budget constraint. Accordingly, we have a contradiction for the first case.

Now turn to the second case $\left(\hat{c}_{j}^{0}>\hat{c}_{j}^{0}\right.$ for some $\left.j\right)$. By $k_{j}{ }^{\prime} s$ 
budget constraint, either $\overline{\mathrm{T}}_{j}>\hat{T}_{j}$, or $\bar{t}_{j-1}>\hat{t}_{j-1}$. without loss of generality, assume $\bar{T}_{j}>\hat{T}_{j}$. We have already demonstrated above that $\bar{c}_{j}^{0}>\hat{c}_{j}^{0}$ and $\vec{T}_{j}>\hat{T}_{j}$ implies $\bar{c}_{j}^{0}>\hat{c}_{j}^{0}$. This returns us to the first case, which yields a contradiction.

The preceding argument suffices to establish that if $\alpha=0$ and $\pi=0$, all BNE yield the same allocation of consumption. Now suppose that $\alpha>0$ or $\pi>0$. Let $\left(C_{i}^{O}, c_{i}^{O}\right)_{i=1}^{N}$ denote the unique BNE consumption profile.

Suppose first that $C_{j}^{0}>0$ for some j. Then efther (i) $T_{j}>0$ and

$$
u^{\prime}\left(C_{j}^{0}\right)=\beta(1-\pi) u^{\prime}\left(c_{j}^{0}\right)+\beta \pi u^{\prime}\left(w_{j}+T_{j}\right)+\alpha v^{\prime}\left(T_{j}\right),
$$

or (ii) $T_{j}=0$ and

$$
u^{\prime}\left(c_{j}^{0}\right) \geqslant \beta(1-\pi) u^{\prime}\left(c_{j}^{0}\right)+\beta \pi u^{\prime}\left(w_{j}\right)+\alpha v^{\prime}(0)
$$

By strict concevity of $u$ and $v$, only one of these conditions can hold; furthermore, (i) can hold for at most one value of $T_{j}$. Thus, $T_{j}$ is uniquely determined. We obtain $t_{j}$ from $p_{j}^{\prime} s$ budget constraint. Now procede by induction. Suppose we know $t_{m}$. Then we obtain $T$ from $k_{m+1}$ 's budget constraint. Knowing $I_{m+1}$, we can calculate $t_{m+1}$ from $p_{m+1}$ 's budget constraint. Applying induction, we conclude that all transfers are uniquely determined.

Next, suppose that $c_{j}^{\circ}=0$ for all $j$. Consider any $i$. Since $T_{i}+t_{i}=w_{i}$, either (i) $0<T_{i}<W_{i}$ and

$$
\begin{aligned}
(1-\pi) u^{\prime}\left(c_{i}^{0}\right) & +\pi u^{\prime}\left(w_{i}+T_{i}\right)+\alpha v^{\prime}\left(T_{i}\right)=(1-\pi) u^{\prime}\left(c_{i+1}^{0}\right)+ \\
& +\pi u^{\prime}\left(w^{i+1}+w^{i}-T^{i}\right)+\alpha v^{\prime}\left(W^{i}-T^{1}\right),
\end{aligned}
$$


$-52-$

or (ii) $T_{i}=0$ and

$$
\begin{aligned}
(1-\pi) u^{\prime}\left(c_{i}^{0}\right)+\pi u^{\prime}\left(w_{i}\right) & +\alpha v^{\prime}(0) \leqq(1-\pi) u^{\prime}\left(c_{i+1}^{0}\right)+ \\
& +\pi u^{\prime}\left(w_{i+1}+w_{i}\right)+\alpha v^{\prime}\left(w_{i}\right),
\end{aligned}
$$

or (iii) $T_{i}=W_{i}$ and

$$
\begin{gathered}
(1-\pi) u^{\prime}\left(c_{i}^{0}\right)+\pi u^{\prime}\left(w_{i}+w_{i}\right)+\alpha v^{\prime}\left(w_{i}\right) \geqq(1-\pi) u^{\prime}\left(c_{i+1}^{0}\right)+ \\
+\pi u^{\prime}\left(w_{i+1}\right)-+\alpha v^{\prime}(0) .
\end{gathered}
$$

By strict concavity of $u$ and $v$, only one of these three conditions can hold; furthermore, (i) can hold for at most one value of $T_{i}$.

Thus, $T_{i}$ is uniquely determined for each $i$, as is $t_{i}\left(t_{i}=W_{i}-T_{i}\right)$.

Q.E.D.

Proofs of Propositions 1, 2, and 3 given in the text.

Theorem 4: The formula for $\lambda+\lambda^{-1}$ follows directly from substitution into (A17a). Without loss of generality, take $k=1$. Then from $(A 32)$,

$$
\begin{aligned}
\frac{d C_{1}}{d W_{1}}= & 1-\frac{d T_{1}}{d W_{1}}-\frac{d t_{1}}{d W_{1}} \\
= & 1-2\left(1-\lambda^{N}\right)^{-1}\left(\lambda-\lambda^{-1}\right)^{-1}\left[\lambda+\lambda^{N-1}-\left(1+\frac{\alpha v^{\prime \prime}(T)}{\beta u^{\prime \prime}(c)}\right)\left(1+\lambda^{N}\right)\right] \\
= & 1-2\left(1-\lambda^{N}\right)^{-1}\left(\lambda-\lambda^{-1}\right)^{-1}\left[\lambda+\lambda^{N-1}-\left(1+\lambda^{N}\right)\left(\lambda+\lambda^{-1}\right) / 2\right. \\
& \left.\quad-\left(1+\frac{\alpha v^{\prime \prime}(T)}{\beta u^{\prime \prime}(c)}-\left(\lambda+\lambda^{-1}\right) / 2\right)\left(1+\lambda^{N}\right)\right] \\
= & 1-2\left(1-\lambda^{N}\right)^{-1}\left(\lambda-\lambda^{-1}\right)^{-1}\left[\left(\lambda-\lambda^{-1}+\lambda^{N-1}-\lambda^{N+1}\right) / 2\right.
\end{aligned}
$$




$$
\begin{aligned}
& \left.+\left(\frac{\alpha v^{\prime \prime}(T)}{u^{\prime \prime}(C)}+\frac{\left[\alpha v^{\prime \prime}(T)\right]^{2}}{2 \beta u^{\prime \prime}(C) u^{\prime \prime}(c)}\right)\left(1+\lambda^{N}\right)\right] \\
=1-2(1 & \left.-\lambda^{N}\right)^{-1}\left(\lambda-\lambda^{-1}\right)^{-1}\left[\left(1-\lambda^{N}\right)\left(\lambda-\lambda^{-1}\right) / 2\right. \\
& \left.+\frac{\alpha v^{\prime \prime}(T)}{u^{\prime \prime}(C)}\left(1+\frac{\alpha v^{\prime \prime}(T)}{2 \beta u^{\prime \prime}(c)}\right)\left(1+\lambda^{H}\right)\right] \\
= & \left(1+\lambda^{N)}\right)\left(1-\lambda^{N}\right)^{-1}\left(\lambda^{-1}-\lambda\right)^{-1} \frac{\alpha v^{\prime \prime}(T)}{u^{\prime \prime}(C)}\left(2+\frac{\alpha v^{\prime \prime}(T)}{\beta u n(c)}\right)
\end{aligned}
$$

as desired. Fron $(A 31)$. we have

$$
\begin{aligned}
\frac{d C_{j}}{d W_{1}} & =-\frac{d T_{1}}{d W_{1}}-\frac{d t}{d W_{1}} \\
& =\left(1-\lambda^{N}\right)^{-1}\left(\lambda^{-1}-\lambda\right)^{-1}\left[\lambda^{j}+\lambda^{j-2}+\lambda^{N-j+2}+\lambda^{N-j}\right. \\
& =\left(1-2\left(1+\frac{\alpha v^{n}(T)}{\beta u^{\prime \prime}(c)}\right)\left(\lambda^{j-1}+\lambda^{N-j-1}\right)\right]\left(\lambda^{-1}-\lambda\right)^{-1}\left[\lambda+\lambda^{-1}-2\left(1+\frac{\alpha v^{n}(I)}{\beta u^{\prime \prime}(c)}\right)\left(\lambda^{j-1}+\lambda^{N-j+1}\right)\right. \\
& =\left(\lambda^{j-1}+\lambda^{N-j+1}\right)\left(1-\lambda^{N}\right)^{-1}\left(\lambda^{-1}-\lambda\right)^{-1} \frac{\alpha v^{n}(T)}{u^{n}(C)}\left(2+\frac{\alpha v^{n}(I)}{\beta u^{\prime \prime}(c)}\right)
\end{aligned}
$$

Finally, note that the labelling of parents is arbitrary, so that we can always relabel to make any given parent $p_{1}$, and either of the parents With whom he shares his children $p_{2} \cdot$ Relabelling produces the desired formula.

Proposition 4: By Theorems 2 and 3, for all symmetric endowment levels $w$ and $w$, the unique equilibrium is symmetric. It can therefore be characterized by the first order condition

$$
u^{\prime}(C)-\beta u^{\prime}(w+w-C)-\sigma v^{\prime}\left(\frac{w-C}{2}\right)=0
$$


Since $u$ and $v$ are concave, the equilibrium value of $C$ is also the solution to

$$
\max _{\substack{0<C<W}} u(C)+\beta u(w+w-C)+2 \alpha v\left(\frac{w-C}{2}\right)
$$

(note that this yields (C1) as the first order condition). Since this problem satisfies all the hypotheses of the maximum theorem, its solution is continuous in $a$. Let $c^{0}$ denote the equilibrium value for $z=0$ (by assumption $0<c^{0}<w$ ); let $c^{0} \equiv w+w-C^{0}$ and $T^{0}=\left(W-C^{0}\right) / 2$.

From (C1), it follows that

$$
\frac{d C}{d \delta}=\frac{\frac{\alpha}{2} v^{\prime \prime}(T)}{u^{\prime \prime}(C)+\beta u^{\prime \prime}(c)+\frac{\alpha}{2} v^{\prime \prime}(T)}
$$

Letting $\alpha \rightarrow 0$ and noting that $C \rightarrow C^{0}, c \rightarrow c^{0}$, and $T \rightarrow T^{0}$ with $C^{0}, C^{0}, T^{0}>0$, we immediately have $\lim _{\alpha \rightarrow 0} \frac{d C}{d \delta}=0$. Since $c=w+W-C$, We also have $\lim _{\alpha \rightarrow 0} \frac{d c}{d \delta}=0$. Thus, $\lim _{\substack{\alpha \rightarrow 0\\}} R^{\delta}=0$. Next, from Theorem 4 we have

$$
\begin{aligned}
& \lim _{\alpha \rightarrow 0} \frac{d C_{1}}{d W_{i}}=\lim _{\alpha \rightarrow 0} {\left[\left(1-\lambda^{N}\right)^{-1}\left(\lambda^{-1}-\lambda\right)^{-1} \alpha\right] } \\
& \cdot\left[\left(\lambda^{i-1}+\lambda^{N-i+1}\right) \frac{v^{\prime \prime}(T)}{u^{\prime \prime}(C)}\left(2+\frac{\alpha v^{\prime \prime}(T)}{\beta u^{\prime \prime}(c)}\right)\right]
\end{aligned}
$$

We have written this as the limit of the product of two expressions. Since $\lim \lambda=1$, the limit of the second expression is $4 v^{\prime \prime}(T) / u^{\prime \prime}(C)$. $\alpha \rightarrow 0$

Note that this limit does not depend upon i. Furthermore, the first 
As in the proof of Theorem 4, relabelling produces the desired formula for the derivatives with respect to $w_{k}$

Proposition 8: Equilibrium is characterized by the set of first order conditions

$$
\beta\left[u^{\prime}\left(w_{i}+T_{i+1}+T_{i}\right)+u^{\prime}\left(w_{i+1}+T_{i+1}+T_{i}\right)\right]=2 u^{\prime}\left(w_{i}-2 T_{i}\right)
$$

Implicitly differentiating these conditions with respect to $\delta$ yields

$$
\begin{aligned}
& -2 u^{\prime \prime}\left(c_{i}\right)+\beta\left[u^{\prime \prime}\left(c_{i}\right)+u^{\prime \prime}\left(c_{i+1}\right)\right] \\
& =4 u^{\prime \prime}\left(c_{i}\right) \frac{d T_{i}}{d \delta}+\beta\left[u^{\prime \prime}\left(c_{i}\right)\left(\frac{d T_{i}-1}{d \delta}+\frac{d T_{i}}{d \delta}\right)+u^{\prime \prime}\left(c_{i+1}\right)\left(\frac{d T_{i}}{d \delta}+\frac{d T_{i+1}}{d \delta}\right)\right] .
\end{aligned}
$$

There are $N$ such equations in $N$ unknowns (the $d T_{i} / d \delta$ ). one can verify by inspection that

$$
\frac{d T}{d \delta}=\frac{1}{2}
$$

for all $i$ satisfies these equations. From this, it is trivial to verify the desired result. Q.E.D.

\section{Proposition 9}

Part 1: $\lim _{N \rightarrow \infty} \frac{\mathrm{dC}_{1}}{\mathrm{~d} \tau_{1}}=\lim _{\mathrm{N} \rightarrow \infty} \frac{\mathrm{dC}_{1}}{\mathrm{dW}_{i}}>0$

\section{Recall that}

$$
\frac{d C_{i}}{d \tau_{i}}=(N-1)^{-1}\left[N \frac{d C_{1}}{d W_{i}}-\frac{d C}{d W}\right]
$$


Since the right hand side is positive and finite, we have the desired conclusion.

Q.E.D.

\section{Proposition 5}

Part 1: $\lim _{\substack{\mathrm{N} \rightarrow \infty \\ \mathrm{R}^{\tau}}}{ }^{\mathrm{i}}=1$

Without loss of generality, take $i=1$. First, we prove that $\lim R^{\tau_{1}} \geq 1$. For any sequence $N_{k} \rightarrow \infty$, choose another sequence of $n \rightarrow \infty$

positive integers $M_{k} \rightarrow \infty$ with $-M_{k} / N_{k} \rightarrow 0$. Define

$$
\begin{aligned}
\left.P_{k}=-i \mid 1 \leq i \leq M_{k} \text { or } N_{k}-M_{k}+2 \leq i \leq N_{k}\right\} \\
\left.k_{k}=1 i \mid 1 \leq i \leq M_{k} \text { or } N_{k}-N_{k}+3 \leq i \leq N_{k}\right\}
\end{aligned}
$$

Note that

$$
\begin{aligned}
& \left.R^{\tau} 1=\sum_{i=1}^{N}\left(\left|\frac{d C_{i}}{d \tau_{1}}\right|\right)+\left|\frac{d c_{i}}{d \tau_{1}}\right|\right) / 2 \\
& \geq\left|\sum_{i \in P_{k}} \frac{d C_{i}}{d \tau_{1}}+\sum_{i \in K_{k}} \frac{d c_{i}}{d \tau_{1}}\right| / 2+\left|\sum_{i \neq P_{k}} \frac{d C_{i}}{d \tau_{1}}+\sum_{i \in K_{k}} \frac{d c_{i}}{d \tau_{1}}\right| / 2 \\
& =\mid \sum_{i \in E_{k}} \frac{d C_{i}}{d \tau_{1}}+\sum_{i \varepsilon K_{k}} \frac{d c_{i}}{d \tau_{1}}
\end{aligned}
$$

(where the final equality follows from the fact that $\tau_{1}$ does not alter agregate consumption). Noting that

$$
\begin{aligned}
\frac{d C_{i}}{d \tau_{1}} & =\frac{d C_{i}}{d W_{1}}-\sum_{j=2}^{N}(N-1)^{-1} \frac{d C_{i}}{d W_{j}} \\
& =(N-1)^{-1}\left[N \frac{d C_{i}}{d W_{1}}-\sum_{j=1}^{N} \frac{d C_{i}}{d W_{j}}\right]
\end{aligned}
$$




$$
=(N-1)^{-1}\left[N \frac{d C_{i}}{d w_{1}}-\frac{d C}{d w}\right]
$$

along with a similar expression for $\frac{d c_{i}}{d \tau_{1}}$, and using symmetry around $p_{1}$, we have

$$
\begin{aligned}
& R^{\tau}+\geq\left(N_{k}-1\right)^{-1} \mid N_{k}\left[\frac{d C_{1}}{d w_{1}}+2 \sum_{i=2}^{k_{k}}\left(\frac{d C_{i}}{d w_{1}}+\frac{d c_{i}}{d w_{1}}\right)\right] \\
& \left.-\left(2 m_{k}-2\right)\left(\frac{d C}{d w}+\frac{d c}{d w}\right)-\frac{d C}{d W}\right) \\
& =(N-1)^{-1} \mid N_{k}\left[\frac{d C_{1}}{d W_{1}}+2 \sum_{i=2}^{M_{k}}\left\{\left(\frac{d T_{i}}{d W_{1}}+\frac{d t_{i}-1}{d W_{1}}\right)-\left(\frac{d T_{1}}{d W_{1}}+\frac{d t_{1}}{d W_{1}}\right)\right\}\right] \\
& -\left(2 M_{k}-2\right)-\frac{d C}{d w} \\
& =\left(N_{k}-1\right)^{-1}\left|N_{k}\left[\frac{d C_{1}}{d W_{1}}+2 \frac{d t_{1}}{d W_{1}}-2 \frac{d t_{W_{1}}}{d W_{1}}\right]-\left(2 M_{k}-2\right)-\frac{d C}{d w}\right| \\
& =\left(N_{k}-1\right)^{-1}\left|N_{k}\left[1-2 \frac{d t_{k}}{d w_{1}}\right]-\left(2 M_{k}-2\right)-\frac{d C}{d w}\right|,
\end{aligned}
$$

where the last equality follows from the fact that $\frac{d t_{1}}{d W_{1}}=\frac{d T_{1}}{d W_{1}}$, and $C_{1}+T_{1}+t_{1}=w_{1}$.

Now we take limits. Recall that the symmetric equilibrium allocation is independent of $N$. From (A31), it is obvious that since $M_{k} \rightarrow \infty$ and $N_{k}-M_{k}+\infty$ as $k \rightarrow \infty$, then $\frac{d t_{k}}{d w_{1}}+0$ (given $\left.0<\lambda<1\right)$. Further, $\left(N_{k}-1\right)^{-1} \rightarrow 0, N_{k}\left(N_{k}-1\right)^{-1} \rightarrow 1$, and $\left(N_{k}-1\right)^{-1}\left(M_{k}-1\right) \rightarrow 0$. He are therefore left with $\lim _{N \rightarrow \infty} R^{\tau} \geq 1$, as desired. 


$$
\begin{aligned}
& \text { Now we a rgue that } \lim _{N \rightarrow \infty} R^{\tau} \leqq 1 \\
& \begin{aligned}
R^{\tau}{ }^{1} & =\sum_{i=1}^{N}\left(\left|\frac{d C_{i}}{d \tau_{1}}\right|+\left|\frac{d c_{i}}{d \tau_{1}}\right|\right) / 2 \\
& =(N-1)^{-1} \sum_{i=1}^{N}\left(\left|N \frac{d C_{i}}{d W_{1}}-\frac{d C_{1}}{d W}+\right| N \frac{d c_{i}}{d W_{1}}-\frac{d c}{d W} \mid\right) / 2 \\
& \leq(N-1)^{-1}\left\{\sum_{i=1}^{N} N\left(\left|\frac{d C_{i}}{d W_{1}}\right|+\left|\frac{i}{d W_{1}}\right|\right)+\sum_{i=1}^{N}\left(\left|\frac{d C}{d W}\right|+\left|\frac{d C}{d W}\right|\right) \mid / 2\right.
\end{aligned}
\end{aligned}
$$

From Theorem 4, it is clear that $\frac{d C_{1}}{d W_{1}}>0$ for all 1. It follows that $\frac{d}{d w_{1}}>0^{-}$for all $i$, otherwise the first order condition for some parent. would be violated. Furthermore, from the derivation in the proof of proposition 4 , it is clear that $\frac{d C}{d w}>0$ and $\frac{d c}{d w}>0$. Thus,

$$
\begin{aligned}
R^{\tau_{1}} & \leqq(N-1)^{-1}\left[\sum_{i=1}^{N} N\left(\frac{d C_{i}}{d w_{1}}+\frac{d c_{i}}{d w_{i}}\right)+N\left(\frac{d C}{d W}+\frac{d c}{d W}\right)\right\} / 2 \\
& =(N-1)^{-1} N
\end{aligned}
$$

From this, it is immediately clear that $\lim _{\mathbb{N} \rightarrow \infty} \mathrm{R}^{\tau} \leqq 1$.

Part 2: $\lim _{N \rightarrow \infty} \frac{d C_{i}}{d \tau_{i}}=\lim _{N \rightarrow \infty} \frac{d C_{i}}{d W_{i}}>0$

As before, without loss of generality, take $i=1$. We know that

$$
\frac{d C_{1}}{d \tau_{1}}=(N-1)^{-1}\left[N \frac{d C_{1}}{d w_{1}}-\frac{d C}{d w}\right]
$$

Since the symmetric equilibrium allocation is independent of $N$, the second term disappears in the limi:. The first term converges to $\frac{d C_{1}}{d w_{1}}$, as desired. Finally, using Theorem 4, 


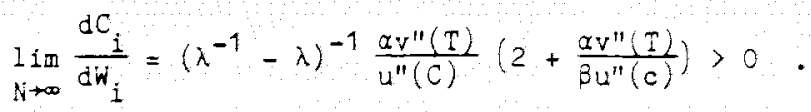

O.E.D.

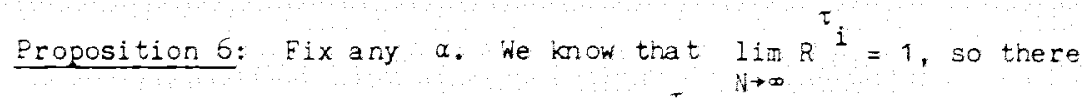
exists $N_{\alpha}$ such that for ali $N>N_{\alpha},\left|1-R^{\tau}\right|<\alpha$. Eor each $a$, let $\hat{N}(\alpha)=N_{\alpha}$. Consider sone sequence $\left(\alpha_{k}, N_{k}\right) \rightarrow(0, \infty)$ as $k \rightarrow \infty$ with $N_{k} \geq \hat{N}\left(\alpha_{k}\right)$ for all $k$. Then, for each $k,\left|1-R{ }^{i}\right|<a_{k}$. Since $\alpha_{k} \rightarrow 0, R^{\tau} \rightarrow 1$. Eurther, $R^{\delta}$ is independent of $N$, so $R^{\delta} \rightarrow 0$ follows from proposition 4 .

\section{Eroposition 7: By Theoren 4,}

$$
\frac{d C_{i}}{d W_{i}}=\left[\left(1-\lambda^{N}\right)^{-1}\left(\lambda^{-1}-\lambda\right)^{-1} \alpha\right]\left[\frac{v^{\prime \prime}(T)}{u^{\prime \prime}(C)}\left(2+\frac{\alpha v^{\prime \prime}(T)}{\beta u^{\prime \prime}(c)}\right)\left(1+\lambda^{N}\right)\right] .
$$

From this formula, it is possible to deduce the following three properties:

$$
\begin{aligned}
& \text { (i) } \frac{d C_{i}}{d W_{i}}>0 \\
& \text { (ii) } \frac{d C_{i}}{d W_{i}} \text { is decreasing in } N \\
& \text { (iii) } \lim _{a \rightarrow 0} \frac{d C_{i}}{d W_{i}}<N^{-1}
\end{aligned}
$$

Property (i) is straightforward to check. Propeerty (ii) follows from the fact that $\left(1-\lambda^{N}\right)^{-1}\left(1+\lambda^{N}\right)$ is decreasing in $N$. We establish property (iii) as follows.

In the proof of proposition 4, we showed that 


$$
\lim _{a \rightarrow 0}\left[\left(1-\lambda^{N}\right)^{-1}\left(\lambda^{-1}-\lambda\right)^{-1} a\right]=\left[\frac{\beta u^{\prime \prime}\left(c^{0}\right)}{u^{\prime \prime}\left(c^{0}\right)+\beta u^{\prime \prime}\left(c^{0}\right)}\right]\left[4 N \frac{v^{n}\left(T^{0}\right)}{u^{\prime \prime}\left(c^{0}\right)}\right]^{-1}
$$

Thus,

$$
\begin{aligned}
\lim _{a \rightarrow 0} \frac{d C_{i}}{d w_{i}} & =\left[\frac{\beta u^{\prime \prime}\left(c^{0}\right)}{u^{\prime \prime}\left(C^{0}\right)+\beta u^{\prime \prime}\left(c^{0}\right)}\right]\left[4 n \frac{v^{\prime \prime}\left(T^{0}\right)}{u^{\prime \prime}\left(C^{0}\right)}\right]^{-1}\left[4 \frac{v^{\prime \prime}\left(T^{0}\right)}{u^{\prime \prime}\left(C^{0}\right)}\right] \\
& =N^{-1}\left[\frac{\beta u^{\prime \prime}\left(c^{0}\right)}{u^{\prime \prime}\left(C^{0}\right)+\beta u^{\prime \prime}\left(c^{0}\right)}\right]<N^{-1} .
\end{aligned}
$$

Now suppose that the proposition is false. - Then by property (i), there must exist a sequence $\left\langle\alpha_{k}, N_{k}\right\rangle_{k=1}^{\infty}$ converging to $(0, \infty)$ such that

$$
\left(\frac{d C_{1}}{d W_{1}}\right)_{k}>n>0
$$

for all k. Ey Proposition 3, we can without loss of generality take $a_{k}>0$ for all k. Choose $k^{*}$ such that

$$
\mathrm{iv}_{\mathrm{K}^{*}}^{-1}<\eta-\varepsilon
$$

for some $\varepsilon>0$. Consider a subsequence $k_{p}$ such that $N_{k}>N_{k^{*}}$ for all p. Then, by property (ii),

$$
\left.\left.\frac{d C_{1}}{d w_{1}}\right|_{N_{k}^{*}, \alpha_{k_{p}}>\frac{d C_{1}}{d w_{1}}}\right|_{N_{p}}, \alpha_{k_{p}}>\eta>N_{k^{*}}^{-1}+\varepsilon
$$

so

$$
\left.\lim _{p \rightarrow \infty} \frac{d C_{1}}{d W_{1}}\right|_{K^{*}}, \alpha_{k p} \geq N_{k^{*}}^{-1}+\varepsilon
$$

But this contradicts property (iii). Q.E.D. 
Theorem 5: Each player's strategy set is the interval $\left[0, w_{i} / 2\right]$ (he chooses a transfer belonging to this interval). Obviously, this is compact and convex. Further, $F_{i}$ 's utility is continuous, and concave in $\mathrm{T}_{1}$. As in Theorem 1, we immediately have existence.

Next, note that if we have an equilibrium where $t_{i}=I_{i}$ for al1 $i$ without imposing this as a constraint, this configuration remains an equilibrium when the constraint is imposed, since the effect of this is only to limit deviations. Thus, the existence of a symmetric equilibrium when endownts are symetric is guranteed by Theorem 2.

Einally, we come to uniqueness. Throughout our argument, we will refer to parents first order conditions, which, for an interior solution, can be written as

$$
2 u^{\prime}\left(c_{j}\right)=\beta\left(u^{\prime}\left(c_{j}\right)+u^{\prime}\left(c_{j+1}\right)\right)
$$

Now suppose, contrary to the theorem, that there are two distinct equilibria, $\left(\bar{T}_{i}\right)_{i=1}^{N}$ and $\left(\hat{T}_{i}\right)_{i=1}^{N}$. Then, wi thout loss of generality, there exists some $i$ for which $\vec{q}_{i}>\hat{T}_{i}$. By $p_{1}$ 's budget constraint, $\vec{C}_{i}<\hat{C}_{i}$ Inspection of $p_{i}^{\prime}$ s first order condition (recalling that $u$ is strictly concave) reveals that either $\bar{c}_{i}<\hat{c}_{i}$, or $\bar{c}_{i+1}<\hat{c}_{i+1}$ Without loss of generality, assume the latter. Then by $k_{i+1}$ 's budget constraint, $\overline{\mathrm{T}}_{i+1}<\hat{\mathrm{T}}_{i+1}$, and $\overline{\mathrm{T}}_{i}-\hat{\mathrm{T}}_{i}<\hat{\mathrm{T}}_{i+1}-\overline{\mathrm{T}}_{i+1}$.

Now we procede by induction. Suppose first that $m$ is odd, $\overline{\mathrm{T}}_{i+m}<\hat{T}_{i+m}$, and $\bar{c}_{i+m}<\hat{c}_{i+m}$. Then, by $p_{i+m}$ 's budget constraint, $\bar{c}_{i+m}>\hat{c}_{i+m}$ By $p_{i+m}$ 's first order condition, $\bar{c}_{i+m+1}>\hat{c}_{i+m+1}$. By $k_{i+m+1}$ 's budget constraint, $\bar{T}_{i+m+1}>\hat{T}_{i+m+1}$, and $\bar{T}_{i+m+1}-\hat{T}_{i+m+1}>$ 
$-62-$

$$
\hat{T}_{i+\mathbb{L}}-\bar{T}_{i+m}=
$$

Suppose that $m$ is even, $\hat{\bar{F}}_{i+m}\left\langle\overline{\mathrm{F}}_{i+m}\right.$, and $\left.\bar{c}_{i+m}\right\rangle \hat{c}_{i+m}$. Then, by $p_{i+m}$ 's budget constraint $\hat{C}_{i+m}>\bar{C}_{i+m}$. By $P_{i+m}$ 's first order condition, $c_{i+m+1}>\vec{c}_{i+m+1}$. By $k_{i+m+1}$ 's budget constraint, $\bar{T}_{i+m=1}<\hat{T}_{i+m+1}$, and $\hat{T}_{i+m+1}-\bar{T}_{i+m+1}>\bar{T}_{i+m}-\hat{T}_{i+m}$.

Applying induction, we see that

$$
\left|\overline{\mathrm{T}}_{i+m+1}-\hat{\mathrm{T}}_{i+m+1}\right|>\left|\overline{\mathrm{T}}_{i+m}-\hat{\mathrm{T}}_{i+m}\right|>\ldots>\left|\overline{\mathrm{T}}_{i}-\hat{\mathrm{T}}_{i}\right|
$$

for all $\pi$ : taking $m=\hat{N}$ yields a contradiction.

Q. ED.

Theorem 6: The formula for $\lambda+\lambda^{-1}$ follows directly from substitution into (B12a). From (B17), we have

$$
\begin{aligned}
\frac{d C_{1}}{d W_{1}} & =1-2 \frac{d T_{1}}{d w_{1}} \\
& =1-4 \frac{u^{\prime \prime}(C)}{\beta u^{\prime \prime}(c)}\left(1-\lambda^{N}\right)^{-1}\left(\lambda-\lambda^{-1}\right)^{-1}\left(1+\lambda^{N}\right) \\
& =1+\left(\lambda+\lambda^{-1}+2\right)\left(1-\lambda^{N}\right)^{-1}\left(\lambda-\lambda^{-1}\right)^{-1}\left(1+\lambda^{N}\right) \\
& =1-(1+\lambda)\left(1+\lambda^{N}\right)(1-\lambda)^{-1}\left(1-\lambda^{N}\right)^{-1} \\
& =-2 \lambda\left(1+\lambda^{N-1}\right)(1-\lambda)^{-1}\left(1-\lambda^{N}\right)^{-1}
\end{aligned}
$$

as desired. Also from (B17),

$$
\begin{aligned}
\frac{\mathrm{dC}_{j}}{\mathrm{dW}} & =-2 \frac{\mathrm{dT}}{\mathrm{dW}_{1}} \\
& =-4 \frac{\mathrm{u}^{\prime \prime}(C)}{\beta \mathrm{u}^{\prime \prime}(c)}\left(1-\lambda^{N}\right)^{-1}\left(\lambda-\lambda^{-1}\right)^{-1}\left(\lambda^{j-1}+\lambda^{N-j+1}\right) \\
& =\left(\lambda+\lambda^{-1}+2\right)\left(1-\lambda^{N}\right)^{-1}\left(\lambda-\lambda^{-1}\right)^{-1}\left(\lambda^{j-1}+\lambda^{N-j+1}\right) \\
& =-(1+\lambda)\left(1-\lambda^{N}\right)^{-1}(1-\lambda)^{-1}\left(\lambda^{j-1}+\lambda^{N-j+1}\right) .
\end{aligned}
$$


As in the proof of Theorem 4, relabelling produces the desired formula for the derivatives with respect to $w_{k}$

Proposition 8: Equilibrium is characterized by the set of first order conditions

$$
\beta\left[u^{\prime}\left(w_{i}+T_{i+1}+T_{i}\right)+u^{\prime}\left(w_{i+1}+T_{i+1}+T_{i}\right)\right]=2 u^{\prime}\left(w_{i}-2 T_{i}\right)
$$

Implicitly differentiating these conditions with respect to $\delta$ yields

$$
\begin{aligned}
& -2 u^{\prime \prime}\left(c_{i}\right)+\beta\left[u^{\prime \prime}\left(c_{i}\right)+u^{\prime \prime}\left(c_{i+1}\right)\right] \\
& =4 u^{\prime \prime}\left(c_{i}\right) \frac{d T_{i}}{d \delta}+\beta\left[u^{\prime \prime}\left(c_{i}\right)\left(\frac{d T_{i}-1}{d \delta}+\frac{d T_{i}}{d \delta}\right)+u^{\prime \prime}\left(c_{i+1}\right)\left(\frac{d T_{i}}{d \delta}+\frac{d T_{i+1}}{d \delta}\right)\right] .
\end{aligned}
$$

There are $N$ such equations in $N$ unknowns (the $d T_{i} / d \delta$ ). one can verify by inspection that

$$
\frac{d T}{d \delta}=\frac{1}{2}
$$

for all $i$ satisfies these equations. From this, it is trivial to verify the desired result. Q.E.D.

\section{Proposition 9}

Part 1: $\lim _{N \rightarrow \infty} \frac{\mathrm{dC}_{1}}{\mathrm{~d} \tau_{1}}=\lim _{\mathrm{N} \rightarrow \infty} \frac{\mathrm{dC}_{1}}{\mathrm{dW}_{i}}>0$

\section{Recall that}

$$
\frac{d C_{i}}{d \tau_{i}}=(N-1)^{-1}\left[N \frac{d C_{1}}{d W_{i}}-\frac{d C}{d W}\right]
$$


Since the distribution of endowments is symmetric before and after a change in $W$, the equal division constraint is not binding; thus, the formula for $\mathrm{dC} / \mathrm{dw}$ is exactly as in the proof of Rroposition 4 (taking $\alpha=0):$

$$
\frac{d C}{d W}=\beta u^{\prime \prime}(c)\left[u^{\prime \prime}(C)+\beta u^{\prime \prime}(c)\right]^{-1}
$$

Since $0<\frac{\mathrm{dC}}{\mathrm{dw}}<1$ and since $\mathrm{C}$ ard $\mathrm{C}$ are independent of $\mathrm{N}$, the limit of $(N-1)^{-1} \frac{d C}{d W}$ is 0 . Thus,

$$
\lim _{N \rightarrow \infty} \frac{d C_{i}}{d \tau_{i}}=\lim _{N \rightarrow \infty}(N-1)^{-1} N \frac{d C_{i}}{d W_{i}}=\lim _{N \rightarrow \infty} \frac{d C_{i}}{d W_{i}}
$$

From Theorem 6 , this last term equals $-2 \lambda(1-\lambda)^{-1}$, which is strictly positive as desired.

$$
\text { Part 2: } \operatorname{iim}_{N \rightarrow \infty}^{\tau} R^{\tau}>1
$$

For any $\mathrm{N}_{k}+\infty$ as $k+\infty$, cefine $M_{k}, P_{k}$, and $K_{k}$ as in the proof of Eroposition 5. Note that

$$
\begin{aligned}
R^{\tau_{i}} & =\sum_{i=1}^{N}\left(\left|\frac{d C_{i}}{d \tau_{1}}\right|+\left|\frac{d c_{i}}{d \tau_{1}}\right|\right) / 2 \\
& \geq\left[\sum_{i \in P_{k}}\left|\frac{d C_{i}}{d \tau_{1}}\right|+\sum_{i \varepsilon K_{k}}\left|\frac{d c_{i}}{d \tau_{1}}\right|\right] / 2+\left|\sum_{i \in P_{k}} \frac{d C_{i}}{d \tau_{1}}+\sum_{i k_{k}} \frac{d c_{i}}{d \tau_{1}}\right| / 2 .
\end{aligned}
$$

Consider some $\varepsilon$ satisfying $0<\varepsilon<-2 \lambda(1-\lambda)^{-1}$. By the argument in Part 1 , there exists $N_{1}$ such that for all $N>N_{1}, \frac{d C_{1}}{d \tau_{1}}>\varepsilon$. By an argument similar to that given-in Part 1, it is easy to show that 


$$
\lim _{N \rightarrow \infty} \frac{d C_{3}}{d \tau_{1}}=\lim _{N \rightarrow \infty} \frac{d C_{3}}{d W_{1}}=-\lambda^{2}(1+\lambda)(1-\lambda)^{-1}<0
$$

Consider then some $n$ satisfying $0>n>-\lambda^{2}(1+\lambda)(1-\lambda)^{-1}$. There exists $N_{2}$ such that for all $N>N_{2}, \frac{d C_{3}}{d r_{1}}<n$. Thus, for

$\mathrm{N}>\max \left\{\mathrm{N}_{1}, \mathrm{~N}_{2}\right\}$

$$
R^{\tau} \geqslant\left|\sum_{i \in P_{k}} \frac{d C_{i}}{d \tau}+\sum_{i \in K_{k}} \frac{d c_{i}}{d \tau_{i}}\right| / 2+\left|\sum_{i \in P_{k}} \frac{d C_{i}}{d \tau_{1}}+\sum_{i \in K_{k}} \frac{d c_{i}}{d \tau_{1}}\right| / 2+\min \{\varepsilon, n\}
$$

Proceding exactly as in the proof of eroposition 5, it then follows that for $N>\max \left\{N_{1}, N_{2}\right\}$

$$
R^{\tau}{ }^{1} \geq\left(N_{k}-1\right)^{-1}\left|N_{k}\left[1-2 \frac{d T_{k}}{d W_{1}}\right]-\left(2 M_{k}-2\right)-\frac{d C}{d W}\right|+\min \{\varepsilon, n\}
$$

From $(B 17)$, it is clear that $\frac{d T_{k}}{d W_{1}}+0$ as $k \rightarrow \infty$. Thus,

$$
\lim _{k \rightarrow \infty} R^{\tau} \geqslant 1+\min \{\varepsilon, \eta\}>1
$$

\section{Proposition 10: Note that}

$$
\frac{d T_{j}}{d W_{1}}=-\left(1-\lambda^{N}\right)^{-1}\left(\lambda-\lambda^{-1}\right)-1\left(\lambda+\lambda^{-1}+2\right)\left(\lambda^{j-1}+\lambda^{N-(j-1)}\right) / 2
$$

First, assume that $N$ is even. Then it is easy to check that $d T_{j} / d W_{1}$ is positive if and only if $j$ is odd. Now consider $p_{j}$, with $j \neq 1$ odd. Note that $\mathrm{dT}_{j-1} / \mathrm{dW}_{1}<0$, and $\mathrm{dT}_{j+1} / \mathrm{dw} w_{1}<0$. Recall that $p_{j}$ 's well-being is given by 
$\max _{j} u\left(W-2 T_{j}\right)+\beta\left[u\left(w+T_{j}+T_{j-1}\right)+u\left(w+T_{j}+T_{j+1}\right)\right]$

This is strictly increasing in $T_{j-1}$ and $T_{j+1}$. Thus, $p_{j}$ 's utility must decline as $W_{1}$ rises. We reason analogously for $j$ even.

Next, assume that $N$ is odd. Then it is easy to check that if

$j-1<N-(j-1), d T_{j} / d w_{1}>0$ iff $j$ is odd. Eurther, if

$\mathrm{N}-(j-1)<j-1$, then $d \mathrm{~T}_{j} / \mathrm{d} W_{1}>0$ iff $\mathrm{N}-\mathrm{j}$ is odd. Accordingiy,

if $1 \neq j<N / 2$ then $(j+1)-1<N-[(j+1)-1]$, and so

$d T_{j-1} / d W_{1}$ and $d T_{j+1} / d W_{1}$ are negative iff $j$ is odd. As above, this

implies that $p_{j}$ is worse off; conversely if $j$ is even. If, on the other hand, $N-j+2<N / 2$, then $N-[(j-1)-1]<(j-1)-1$, and $\mathrm{dT}_{j-1} / \mathrm{dw}{ }_{1}$ and $\mathrm{dT} \mathrm{T}_{j+1} / \mathrm{dW}$ are negative iff $\mathrm{N}-j$ is odd. Agin, this implies $p_{j}$ is worse off; conversely if $N-j$ is even. Q.E.D. 


\section{References}

Andreoni, James R. [1986], "Impure Altruisa and Donations to Public Goods: A. Theory of "harm-Gl ow" Giving," mimeo, University of Wisconsin.

Barro, Robert J. [1974], "Are Government Bonds Net Wealth?" Journal of Political Economy 81, 1095-1117.

Bernheir, E. Douglas [1986], "On the Voluntary and Involuntary Provision of Public Goods," American Economic Review 76, 789-793.

Bernheim, B. Douglas and Kyle Bagke11. [1988], "Is Everything Neutral?" Journal of Political Economy $96 ; 308-338$.

Bernheir, B. Douglas, Andrei Shleifer, and Lawrence Sumers [1985], "The Strategic Bequest Motice," Journal of Eolitical Economy 93, 10451076.

Bollabas, B. [1981], "The Dianeter of Random Graphs," Transactions of the American Matheratical Society 267, No. 1.

Debreu, Gerard [1952], "A Social Equilibrium Existence Theorem," Proceedings of the National Academy of Sciences of the U.S.A. 38, $886-893$.

Harsanyi, John [1967-68], "Games with Incomplete Information Played by Bayesian Players," Management Science 14, 159-82, 320-34, 486-502.

Menchik, Paul L. [1980], "Primogeniture, Equal Sharing, and the U.S. Distribution of Wealth," Quarterly Journal of Economic 94, 299-316. 
Figure 1: The structure of Intrafamily linkages

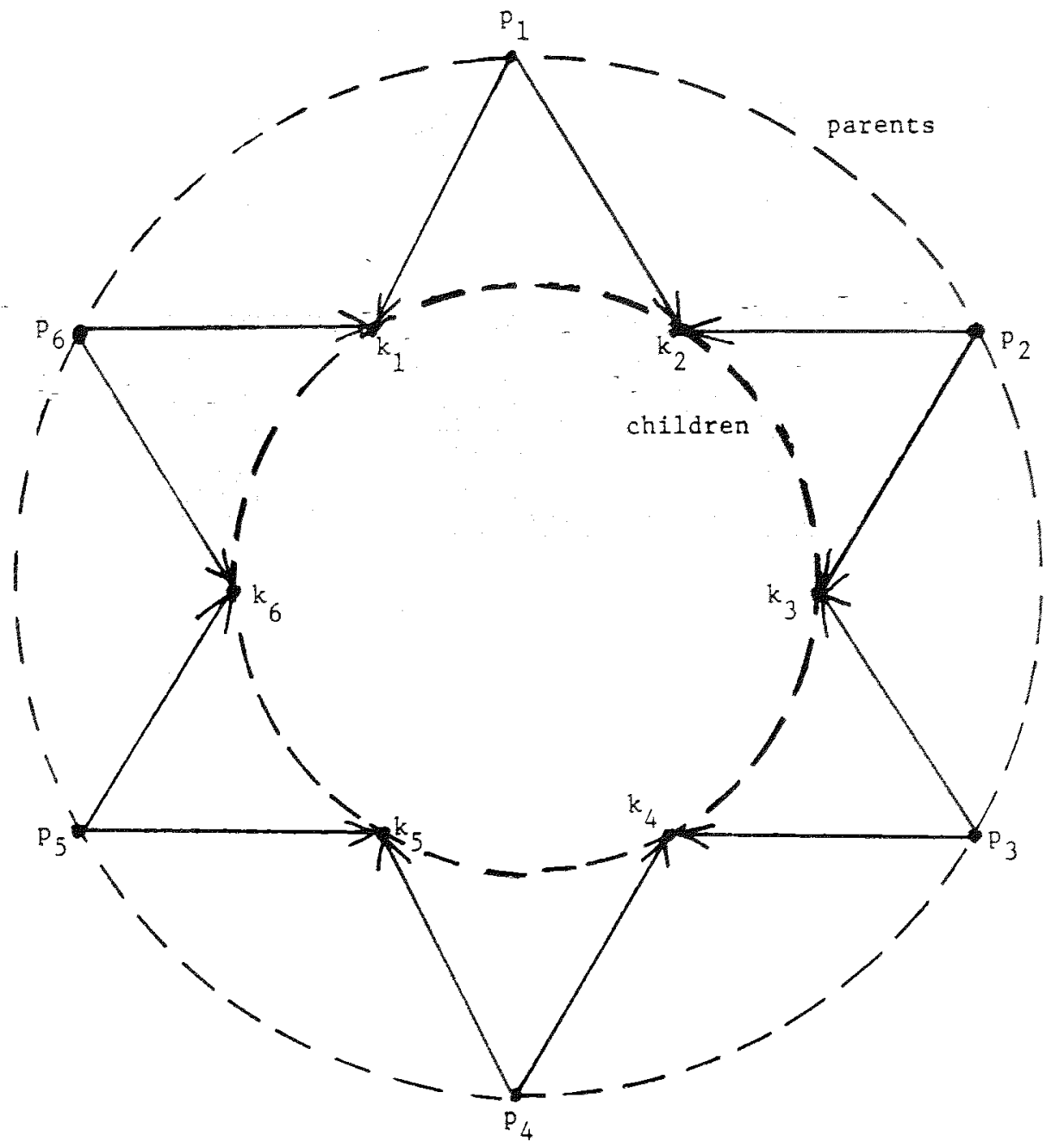


Figure 2: Comparative Statics wtth Friction

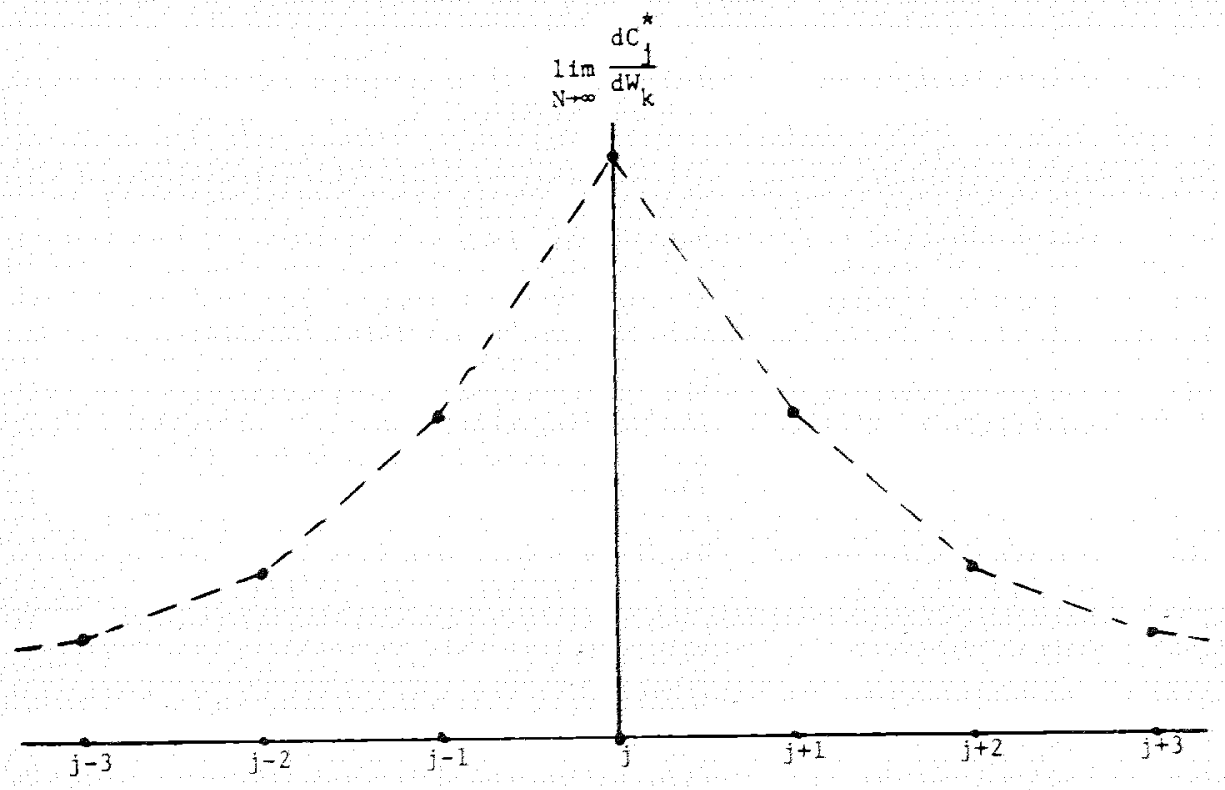

Eigure 3: Comparative Staties with Egalitanarianism

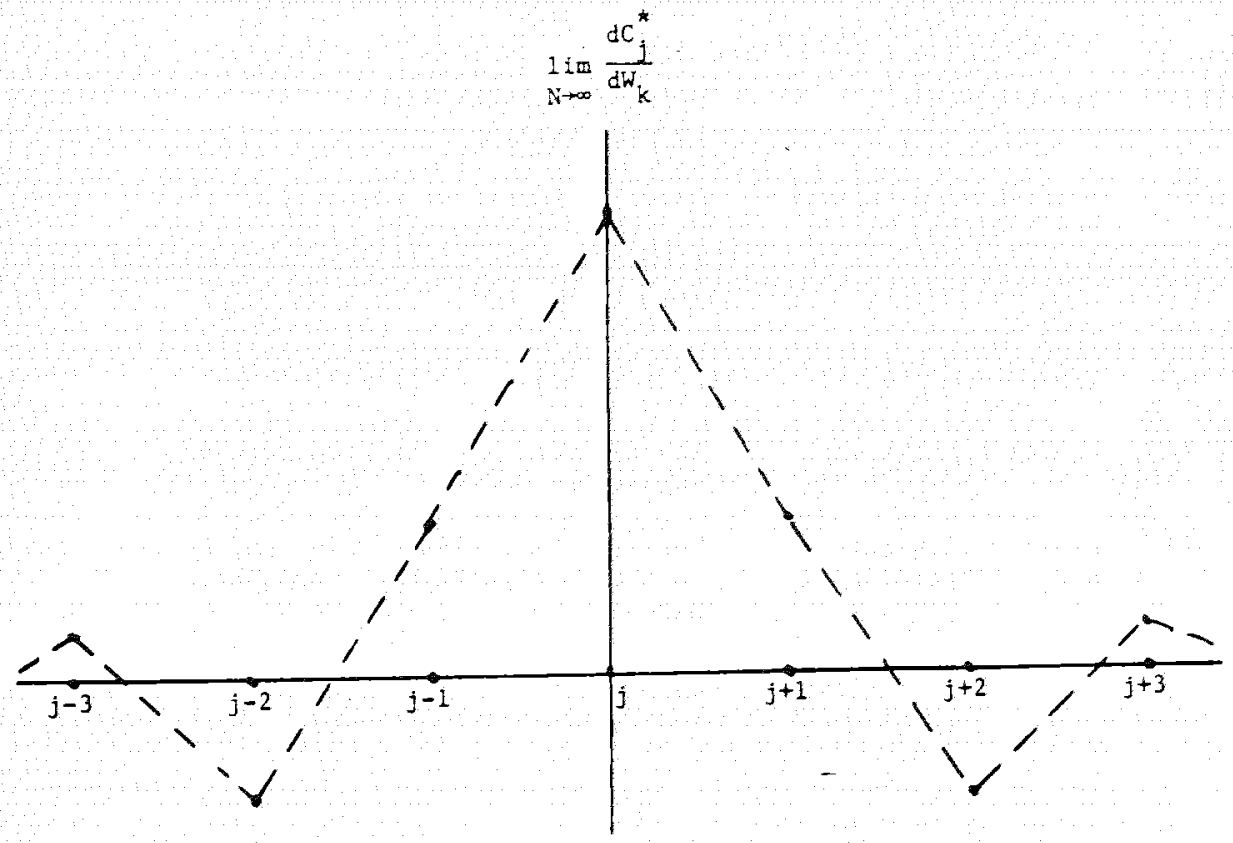

